\title{
The effects of duration, area, and background intensity on the visual intensity difference threshold given by the forced-choice procedure: Derivations from a statistical decision model for sensory discrimination
}

\author{
MICHEL TREISMAN, ${ }^{2}$ UNIVERSITY OF READING, ENGLAND \\ BARRY LESHOWITZ, ${ }^{3}$ BROOKL YN COLLEGE OF THE CITY UNIVERSITY OF NEW YORK
}

\begin{abstract}
A model for visual discrimination is outlined. This assumes that detection is based on separating signal and noise by means of a statistical decision procedure, and that the noise arises from physical and biological sources. When the model is applied to such traditional detection procedures as the method of constant stimuli, it successfully predicts the laws of spatial and temporal summation and the form of the Weber function. The model is extended to the forced-choice procedures. It is found that it predicts departures from the classical laws for spatial and temporal summation and an increase in the slope of the Weber function when the pulsed forced-choice procedure is used, and data are presented that agree with the predictions. It is shown that data obtained with forced choice against a continuous background are consistent with the assumption that the $S$ either covertly detects or fails to detect an increment at each of the two alternative locations and this determines his final overt response, rather than that he directly compares the inputs at the two locations. A comparison between results obtained with the forced-choice procedure with a pedestal, or with the continuous forced-choice procedure, provides further evidence that Ss employ a double detection strategy in the latter case.
\end{abstract}

\section{INTRODUCTION}

At an early stage, the systematic study of sensory discrimination revealed that marked effects on the threshold were produced by such stimulus parameters as background intensity and the duration or area of the stimulus increment. These findings were enshrined as eponymous laws (Weber's law, Bloch's law, Ricco's law, Piper's law, etc.), and although similar effects have been found not only in vision but in other modalities too, the provision of a coherent theoretical treatment to explain and relate the various findings in a single modality, let alone over the range of modalities, has proved a difficult problem.

The way to a possible solution to this problem was opened by the suggestion that repeated presentations of a stimulus of nominally constant intensity will produce effects in the $S$ that vary from trial to trial, and that the quantal variability of light (in the case of visual intensity discrimination to which we shall limit the present discussion) was a source of variation acting to limit discrimination (Thurstone, 1927; Hecht, Shlaer, \& Pirenne, 1942; Rose, 1948; Aguilar \& Stiles, 1954). The problem faced by the sensory mechanisms in making a discrimination was then essentially that of distinguishing a signal from noise, a statistical decision (Tanner \& Swets, 1954; Barlow, 1956; Gregory, 1956; Swets, Tanner, \& Birdsall, 1961).

However, "fluctuation theory" (the name sometimes given to the hypothesis that the threshold is limited by the quantum variability of light) was not wholly successful in explaining the relation between the threshold increment, $\Delta \mathrm{I}$, and the background intensity against which it is measured, I (the relation between $\Delta I$ and I will be referred to as the "Weber function"). If a stimulus background of intensity $I$ is repeatedly presented, the numbers of quanta absorbed by the eye on successive trials will be described by a Poisson distribution, with a mean proportional to $I$ and an SD proportional to $I^{1 / 2}$, which suggests that $\Delta I$ should be proportional to $I^{1 / 2}$. But Aguilar and Stiles (1954) found that the Weber function approximated a square root law only for a limited range of values above the absolute threshold; as the magnitude of $I$ increased beyond this level the data tended towards the linear relation between $\Delta \mathrm{I}$ and $\mathrm{I}$ traditionally known as Weber's law. Barlow (1957) confirmed this finding and asked "can reasons be found for the departures from the theoretical predictions which occur when the test stimulus is of long duration or large area, and when the background intensity is high?"

The relative but not complete failure of the theory suggests that it must be inadequate in some important respect, and what this may be is indicated by the observation that the theory's proponents have almost completely discounted or failed to consider sources of variation other than the physical variability of the stimulus. But if we allow that variability may arise in the nervous pathways as well as in the physical stimulus, it becomes evident that the prediction of a square root law from the quantum variability of light must rest on one of the following assumptions: (1) The discriminatory response is determined at the receptor level, immediately on the absorption of quanta-but there is ample evidence, reviewed by Treisman (1966), that the behavioral threshold is more centrally determined. (2) The absorption of a quantum or quanta triggers a sequence of neural events that result in "messages" being transmitted to the central locus at which the discriminatory response is determined, the magnitude of the total message indicating the size of the stimulus; the variability or "noise" in this central transmission is negligible, so that the variation of the central message from trial to trial reflects only the Poisson variability of the stimulus. But it is almost inconceivable that the processes of impulse generation and transmission across synapses should contribute very much less noise than the limiting quantum variability of light. (3) A message is transmitted centrally, and biological noise does arise during the course of this central transmission, but its characteristics are such as not to alter the form of the law predicted from a consideration of the variability of the stimulus alone (McGill, 1967). This third assumption is tenable but, as we have seen, the square root law that results from it is not consistent with the whole range of experimental findings.

It is apparent that if we wish to determine whether or not a signal-noise theory of sensory discrimination can explain the effects of stimulus parameters on the size of the threshold, we must take into account all the major sources of noise arising in the path from the physical stimulus to the central discriminatory mechanisms. Unfortunately our knowledge of the visual pathways is insufficient for us to use physiological observations to characterize definitively all sources of biological noise. However, Treisman (1964a, 1966) has considered a simplified schema of 


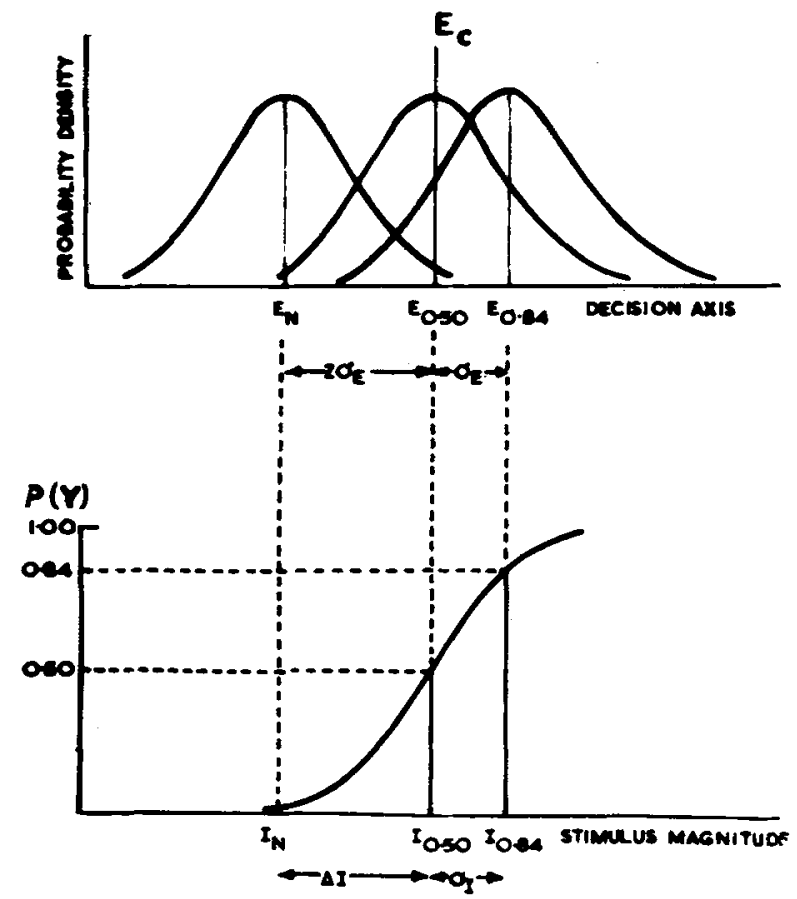

Fig. 1. The relation between the physical stimulus intensity, I, and its central effect, $E$, that underlies measurement of the threshold by detection procedures, such as the method of constant stimuli. The psychometric function given by the MCS relates $P(Y)$, the probability of the response "Yes," to the physical magnitude of the stimuli presented. The three distributions on the central decision axis, $\mathrm{E}$, correspond to three stimuli: $I_{N}$, the background stimulus; $I_{0.50}$, the background + increment whose mean central effect coincides with the criterion $E_{c}$; and $I_{0.84}$, the stimulus whose mean central effect lies 1 SD above $E_{c}$ and which therefore gives $P(Y)=0.84$. The increment threshold for the method of constant stimuli is given by $\Delta I=I_{0.50}-I_{N}$. When $I_{N}=0$ this procedure gives the absolute threshold, which is then $I_{0}=I_{0.50}$.

the visual system that makes minimal assumptions about the physical and biological sources of noise. This model was applied to the increment threshold, the threshold for the detection of a transient increase in the intensity of an area of a uniformly illuminated background field, as determined by such classical procedures as the method of constant stimuli (MCS). It was found that the model successfully predicted the form of the Weber function, giving a square root section at low background intensities with a transition to a linear section as I increased, and it also predicted the form of the spatial summation and the temporal summation curves. Thus, despite the simplification necessarily involved in framing the model, it appeared able to explain a number of different phenomena and to relate them to one another; moreover, the essential ideas are applicable to other modalities, suggesting an explanation for the ubiquity of Weber's law.

The present paper is an attempt to test and develop the model further by applying it to a new area. Treisman (1966) applied it to what may be called the "detection procedures," i.e., those procedures in which the opportunity for an increment occurs once in each trial and the $S$ may detect or fail to detect it. Here we shall apply the model to the "forced-choice procedures" (FCP) in which the increment may occur at two or more alternative locations and the $S$ must decide which one contained it. As we shall see, in this case the predictions differ in some respects from those for the classical procedures, and data are available against which some of these predictions may be tested. But before we consider the forced-choice procedures it will be useful if we first summarize the main assumptions of the model, which is described more fully elsewhere (Treisman, 1964a, 1966), and illustrate how it leads to predictions for the detection procedures.

\section{DETECTION PROCEDURES}

Figure 1 illustrates the assumptions made about the overall strategy of discrimination, when the absolute or increment threshold is determined by such a procedure as the Method of Constant Stimuli. We use I to represent stimulus intensity (e.g., in quanta/sec $\operatorname{deg}^{2}$ ). The constant background field is at intensity $I_{N}$ (when the absolute threshold is to be determined, $I_{N}=0$ ), and one of a set of intensity increments, $\Delta I_{v}$, is added to this on each trial. In the MCS several values of $\Delta \mathrm{I}_{\mathbf{v}}$ are used, presented in a random sequence on successive trials. Each increment is presented for $t_{i}$ sec over an area $A_{i}$ deg $^{2}$. The $S$ is asked to respond "Yes" or "No" on each trial, and for each value of $I_{y}=I_{N}+\Delta I_{y}$ that is used the frequency of the response "Yes," $P(Y)$, is found. A plot of $P(Y)$ against $I$ is called the psychometric function and usually approximates a normal ogive; this is shown in the lower half of Fig. 1. The increment threshold, $\Delta I$, is often taken as $\Delta I=I_{0.50}-I_{N}$, where $I_{0.50}$ is the stimulus such that $\mathrm{P}\left(\mathrm{Y} \mid \mathrm{I}_{0.84}\right)=0.84$. The $\mathrm{SD}$ of the threshold, $\sigma_{\mathrm{I}}$, can be taken as $\sigma_{\mathrm{I}}=\mathrm{I}_{0.84}-\mathrm{I}_{0.50}$.

We suppose that on each trial the stimulus gives rise to a central message, the magnitude of this central effect being represented on a scale or decision axis, E. When the background intensity $I_{N}$ is presented without an added increment, the values of $E$ that result are represented by the "noise" distribution centered on $E_{N}$, the latter being the mean effect of $I_{N}$. Similarly, a series of presentations of any other value of $I$, such as $I_{0.50}$, will give a similar distribution of central effects centered on $E_{0.50}$. As a first approximation we suppose that for the small range of values of $I$ used in determining a threshold these distributions can be taken to be normal with equal variance, $\sigma_{\mathrm{E}}^{2}$. A basic assumption is that the discriminatory mechanisms accumulate statistical information about the values of $E$ that are produced when the background alone is presented, and a positive detection response is given whenever an input departs far enough from the expectations thus built up. This is done by choosing a criterion $E_{c}$ at a value such that the false positive rate $\left[F P R=P\left(Y \mid I_{N}\right)\right]$ does not exceed an acceptable limiting value, \&. [This is the Neyman-Pearson criterion, which seems the most appropriate for the traditional psychophysical procedures (Neyman \& Pearson, 1933; Treisman, 1964b).] Then $\mathrm{E}_{\mathrm{C}}=\mathrm{E}_{\mathrm{N}}+\mathrm{z} \sigma_{\mathrm{E}}$, where $\mathrm{z}$ is the standardized normal deviate corresponding to the limiting false positive rate (thus $\&=0.0227$ would give $\mathrm{z}=2$ ). If on a given trial the input $E$ exceeds $E_{c}$ the $S$ will respond "Yes"; if it does not, "No." It follows that the mean central effect of $I_{0.50}$, which is $E_{0.50}$, will coincide with $E_{c}$, and thus $E_{c}-E_{N}=z \sigma_{E}$ will correspond to $\Delta I$. Similarly, the mean central effect of $\mathrm{I}_{0.84}$ will be $\mathrm{E}_{0.84}$ and thus $\sigma_{1}$ will correspond to $\sigma_{\mathrm{E}}$. The classical procedures have usually been used with well-trained $S$ s giving very small false positive rates. In such cases we might expect $\mathrm{z}$ to be of the order 2-6 (Treisman, 1965).

It appears that in order to make predictions about $\Delta I$ we need to know the determinants of $\sigma_{\mathrm{E}}$, i.e., the sources of noise, and the form of $E=f(I)$, the "transducer function" relating values on the decision axis, $E$, to $I$. This information can be derived from a schema of the visual pathway. For simplicity the pathway is considered to have three stages: (1) the path from the physical 
stimulus source to the retinal receptors; (2) transmission from the receptors across the retina to the ganglion cells; (3) transmission from the ganglion cell to the central locus at which the threshold decision is made.

We shall now describe the schema as it applies to the determination of the threshold by the method of constant stimuli, and we start by considering how the "noise" distribution is built up when the background alone is presented.

(1) The first stage is essentially that of "fluctuation theory." If a stimulus of intensity $I$ and area $A$ is exposed to the eye for duration $t$, the number of quanta absorbed by the receptors is given by fAtI, where $f$ is the fraction of the quanta incident at the cornea that is eventually absorbed by visual pigment. This number is a Poisson variable.

(2) From the receptors to the ganglion cells. The "noise" distribution is built up from samples of the background, which may be of large area and may be exposed for some time. We suppose that the sensory mechanisms take each sample of the retinal input over an area $A_{r}$ and for a duration $t_{r}$, so that the mean number of quantal absorptions falling in a sample of the background is given by $f A_{r} t_{r} I_{N}$. The absorption of quanta of light causes the decomposition of visual pigment molecules, and the transmission of corresponding messages across the retina. Biological noise could arise in two ways: the spontaneous generation of messages that are confusable with those arising from the light stimulus, and variability in the transmission of the visual message along the pathway. It is convenient to consider the former source as though it arose wholly at this stage, as would be the case if it were all caused by the spontaneous decomposition of molecules of visual pigment. It would then be equivalent to the effect of an additional constant background illumination or "dark light," $I_{n}$. Then $m$, the mean number of visual pigment decompositions falling within a sample of the background, is given by $m=f A_{r} t_{r}\left(I_{N}+I_{n}\right)$, and its variance is also equal to $m$.

The intraretinal effects that are triggered by the molecular decompositions are transmitted across the retina and converge on the ganglion cells. We can expect that the mechanisms underlying light adaptation will greatly reduce the volume of messages transmitted from the receptors across the retina, especially when stimulus values are high. Various models for this reduction have been studied by computer simulation (Treisman, 1966); it appears that, for the predictions we are concerned with here, the effects of light adaptation need not be explicitly included. Since there is considerable evidence that transmission through the retina to the ganglion cells is linear (Treisman, 1966), we can take $m$ to represent the convergent effect acting on the ganglion cells to initiate activity in the latter.

(3) Transmission from the ganglion cell to the locus of response determination. It is convenient to consider the output from the ganglion cell as consisting of a number, $\mathrm{m}^{\prime}$, of "sensory messages," each of mean magnitude $s$ (which might correspond, for example, to a constant contribution to the firing of the cell), any nonlinearity in the visual pathway then being contained in the transformation from $m$ to $m^{\prime}$. Then $E_{k}$, the central effect on the decision axis of a given sample $k$, would be the sum of the corresponding $\mathrm{m}_{\mathbf{k}}^{\prime}$ sensory messages,

$$
E_{k}=\sum_{j=1}^{m^{\prime} k} s_{j},
$$

where $s_{j}$ are the individual sensory messages. In this schema the "transducer function," $E=f(I)$, is determined by $m^{\prime}=\cdot(m)$, where $f$ might, for example, be a logarithmic or power function. The computer simulation study provided predictions for various assumptions about the parameters and mechanisms of the model.
When these predictions were compared with data they indicated that the logarithmic function was extremely implausible, but that the results were compatible with existing data provided the transducer function was taken to be a power function with an exponent somewhere in a range of approximately 0.5-1.2 (Treisman, 1966). It is then simplest for the present purpose to consider the case where $m^{\prime}=m$, which gives us, for the expected value of $E_{N}$,

$$
E_{N}=\operatorname{sf}_{\mathbf{r}} t_{\mathbf{r}}\left(I_{N}+I_{n}\right)
$$

This gives the overall functional relation between values on the $E$ and $I$ scales. We now need to derive the variance of distributions on the decision axis, $\sigma_{\mathrm{E}}^{2}$. This arises from the Poisson variability of $m$ (i.e., from the quantum variability of light, and the spontaneous generation of messages or "dark light"), and from the variability attaching to $\mathrm{s}$. This last will arise from variation in the transmission of neural messages along the visual pathway, which will make the magnitude of the "sensory messages" vary about the mean $s$, so that it has a variance $\sigma_{s}^{2}$; we take it that this variation is normal.

Before proceeding to derive $\sigma_{\mathrm{E}}^{2}$ we must consider another relevant parameter, which may be called the intrasample correlation between sensory messages, $r_{s}$. To illustrate this, consider a sequence of $N$ samples, giving central effects $E_{1}, E_{2}$, $\cdots, E_{i}, \cdots, E_{N}$, each being the sum of $m^{\prime}$ sensory messages, and $\mathrm{N}$ being large. Then $E_{i}=s_{i 1}+s_{i 2}+\cdots+s_{i j}+\cdots+s_{i k}+\cdots$ $+s_{\text {im }}$, where of course the second subscripts are assigned to $s$ in an essentially random manner. It follows that the productmoment correlation, $\mathbf{r}_{\mathbf{j k}}$, between any pair of sensory messages $\mathbf{s}_{\mathbf{j}} \mathbf{j}$ and $s_{. k}, j \neq k$, over the $\mathrm{N}$ samples should be the same for all pairs $\mathbf{s}_{\mathrm{j}}$ and $\mathbf{s}_{\mathbf{k}}$ and can therefore be represented as a single value, $\mathbf{r}_{\mathbf{s}}$. Now, whereas the correlation between two random variables can have any value between -1 and +1 , this is not true of $r_{s}$ when $\mathrm{m}^{\prime}>2$, the lower limit then being greater than -1 . It is obvious that when we have three random variables giving $r_{12}=r_{13}=r_{23}=r_{s}$, each variable cannot be correlated at $r_{s}=-1$ with both the others, and in general $r_{0} \leqslant r_{s} \leqslant 1$, where the lower limit is given by $r_{o}=-1 /\left(m^{\prime}-1\right)$. When $m^{\prime}$ is at all large, as will almost always be the case with visual stimuli, $r_{0}$ will be negligibly different from zero. We may also note that there are factors that would tend to make $r_{s}$ substantially greater than this necessary lower limit. For example, variation in the level of oxygenation at different phases of the pulse cycle or other effects of the cardiac and respiratory cycles, or moment-to-moment variation in the level of reticular activation might make the values of the component "sensory messages" summed in a single sample vary for the most part in the same direction from the mean, s. It has been shown that the reaction time is affected by the phase of the pulse cycle (Birren, Cardon, \& Phillips, 1963), as is the amplitude of the EEG (Cohn, 1962), and cortical evoked responses to a visual stimulus are more similar when determined at the same phase of the cardiac or respiratory cycle (Callaway \& Buchsbaum, 1965).

With these assumptions and Eq. 1 we can now write the variance of $E_{N}$ as

$$
\sigma_{E}^{2}=r_{s} \sigma_{s}^{2} f^{2} A_{r}^{2} t_{r}^{2}\left(I_{N}+I_{n}\right)^{2}+\left(s^{2}+\sigma_{S}^{2}\right) f_{A_{r}} t_{r}\left(I_{N}+I_{n}\right)
$$

We saw from Fig. 1 that the increment threshold determined by the MCS is $\Delta I=I_{0.50}-I_{N} \cdot I_{0.50}$ would be the intensity of background plus increment giving a central distribution whose mean coincides with $\mathrm{E}_{\mathrm{c}}$. In considering how the samples that constitute this distribution are made up we must take account of the parameters of sampling for both the background and the increment. We saw that a sample of the background is taken over 
an area $A_{r}$ and duration $t_{r}$. It is simplest to assume that in a trained $S$ these will usually approximate to the area $A_{i}$ and duration $t_{i}$ for which the increment is given. An exception will arise when the physical stimulus area or duration is less than the physiological lower limits on the fineness of the sampling that the visual system is capable of: we suppose that it is not possible for the visual mechanisms to take samples of an area less than $\alpha$ (perhaps corresponding to the size of the receptive field) or duration less than $\tau$. Then in general $A_{r}=\max \left(A_{i}, \alpha\right)$ and $t_{r}=\max \left(t_{i}, \tau\right)$, so that $\alpha$ and $\tau$ are minima for $A_{r}$ and $t_{r}$. We can now use the model to derive predictions.

Since $\mathrm{E}_{\mathrm{c}}=\mathrm{E}_{0.50}$, we can write $\mathrm{E}_{\mathrm{c}}-\mathrm{E}_{\mathrm{N}}=\mathrm{z} \sigma_{\mathrm{E}}=$ $\operatorname{sf}\left[A_{r} t_{r}\left(I_{N}+I_{n}\right)+A_{j} t_{i} \Delta I\right]-\operatorname{sfA}_{r} t_{r}\left(I_{N}+I_{n}\right)=\operatorname{sf} A_{i} t_{i} \Delta I$, so that

$$
\begin{aligned}
\Delta I=\frac{z \sigma_{E}}{s f A_{i} t_{i}}=\frac{z}{f A_{i} t_{i}}\left[r _ { s } v ^ { 2 } f ^ { 2 } A _ { r } ^ { 2 } t _ { r } ^ { 2 } \left(I_{N}\right.\right. & \left.+I_{n}\right)^{2} \\
& \left.+\left(1+v^{2}\right) f A_{r} t_{r}\left(I_{N}+I_{n}\right)\right]^{1 / 2}
\end{aligned}
$$

where $v=\sigma_{s} / s$, the coefficient of variation of the sensory message.

Since Eq. 3 expresses the threshold as a function of background intensity and the duration and area of the increment, it predicts the form of the Weber function and of spatial and temporal summation. The predictions are shown more clearly if we simplify the equation. Since it is very likely that $v^{2}<<1$, when $f A_{r} t_{r}\left(I_{N}+I_{n}\right)$ is small the second expression in the square brackets in Eq. 3 will dominate the relation which will then approximate to

$$
\Delta I=\frac{z A_{r}^{1 / 2} t_{r}^{1 / 2}\left(I_{N}+I_{n}\right)^{1 / 2}}{f^{1 / 2} A_{i} t_{i}}
$$

but when $f A_{r} t_{r}\left(I_{N}+I_{n}\right)$ is large the first expression in the square brackets will be the most important and the equation will approximate to

$$
\Delta I=\frac{z r_{s}^{1 / 2} v A_{I} t_{r}\left(I_{N}+I_{n}\right)}{A_{i} t_{i}}
$$

\section{The Effect of Background Intensity}

Let us suppose that $I_{N}$ alone is varied, being increased from zero. When $I_{N}=0$, Eq. 3 gives $I_{o}$, the absolute threshold. As $I_{N}$ is increased above this level, it will at first be negligible as compared with $I_{n}$ so that the threshold will be substantially constant at the absolute threshold level. If we plot $\log \Delta I$ against $\log I_{N}$ we will have a straight line with a slope of zero. At higher values of $I_{N}$ the relation will be described by Eq. 4 , giving a square root law represented by a line with a slope of 0.5 on the log-log plot. At still higher background intensities, Eq. 5 will apply, giving a transition to a linear relation (Weber's law) represented by a straight line with a slope of 1 . These predictions agree, of course, with the experimental result.

It is of interest to consider the effect of the parameter $A_{i}$, the area of the increment, on the Weber function. When Eq. 4 applies, the larger $A_{r}=A_{i}$ is, the smaller will the threshold be. But the larger $A_{r}$ is the larger will $f_{A_{r}} t_{r}\left(I_{N}+I_{n}\right)$ be, and so the transition to Eq. 5 will occur at lower values of $I_{N}$ if $A_{i}$ is increased. Thus when $A_{i}$ is large Weber's law will be approximated sooner, and the Weber functions for different values of $A_{i} \geqslant \alpha$ will converge at high values of $I_{N}$. Corresponding predictions can be made for the effect of $t_{i}$ as a parameter of the Weber function. These effects are shown by the experimental data (Barlow, 1957).

\section{The Effect of Increment Duration}

If $t_{i}$ alone is varied, then when $t_{i} \leqslant \tau, t_{r}=\tau$ and so $t_{i}$ is the only variable on the right-hand side of Eq. 3, so that the equation approximates to $\Delta I=k / t_{i}$, where $k$ is a constant. This is Bloch's law, and if we plot $\log \Delta I$ against $\log t_{i}$ it will give a straight line with a slope of -1 .

If $t_{i}>\tau$ and $f A_{r} t_{r}\left(I_{N}+I_{n}\right)$ is small, Eq. 4 will apply; this can be written as $\Delta I=k^{\prime} / t_{1}^{1 / 2}$, where $k^{\prime}$ is a constant, giving a line with a slope of -0.5 . As $t_{i}$ increases further Eq. 5 will come to apply, making $\Delta I$ constant, so that there will be a transition to a line of zero slope. It is evident that when the parameters $I_{N}$ or $\mathbf{A}_{\mathbf{i}}$ are large then for $t_{i}>\tau$ we will get a more rapid or immediate transition to the relation described by Eq. 5 .

\section{The Effect of Increment Area}

Exactly similar predictions can be derived for variation in $\mathbf{A}_{\mathbf{i}}$.

The predictions above, and other predictions, have been found to match the data surprisingly well (Treisman, 1966), so that although the model that has been outlined here is clearly oversimple as compared with the redoubtable complexities of the system it attempts to explain, it nevertheless appears to have sufficient explanatory force to justify examining and testing it further.

\section{FORCED-CHOICE PROCEDURES}

The forced-choice procedure (FCP) has recently become popular as a method for determining the threshold, and as the relations between the threshold determined in this way and the stimulus parameters may differ in some respects from those obtained using classical procedures, it is of interest to examine whether or not the model described above can be applied successfully to these procedures.

We shall consider here only the forced choice between two alternatives. In this procedure two stimuli are presented on each trial, $I_{N}$ and $I_{V}=I_{N}+\Delta I_{V}$. These might be presented simultaneously in two different spatial locations, positions a and b; or they might be presented in succession, at two indicated times of occurrence, $a$ and $b$. In each case the $S$ is asked to indicate which position or time of occurrence contained the increment by responding " $a$ " or "b," "Left" or "Right," "First" or "Second," etc.

In this procedure there is a further important experimental variable. The background intensity, $I_{N}$, may be continually present during the trial, and the two stimuli are then produced by adding the increment, $\Delta \mathrm{I}_{\mathrm{v}}$ or 0 , to this background at the indicated positions or times of occurrence. We shall call this "forced choice with continuous background," or "continuous forced choice." Or the background field may be dark (i.e., $I_{n}$ alone is present), and $I_{N}$ is presented as a single pulse covering an area $A_{i}$ for a duration $t_{i}$ at one location and $\left(I_{N}+\Delta I_{V}\right)$ is given with the same area and duration, at the other location. Thus a temporal forced-choice trial might consist of: an initial dark interval-presentation of a stimulus of area $A_{i}=1 \mathrm{deg}^{2}$ illuminated at intensity $I_{N}$ for a duration $t_{i}=20 \mathrm{msec}-$ an interstimulus interval (ISI) of 1 sec-presentation of a stimulus of area $A_{i}=1 \mathrm{deg}^{2}$ illuminated at intensity $I_{v}$ for a duration $t_{i}=20$ msec. The $S$ would then indicate which stimulus was the more intense. We shall call this procedure "forced choice with pulsed stimulation," or "pulsed forced choice."

A third procedure, the "pedestal FCP," has features of both the others. A background of intensity $I_{N}$ is continually present during each trial, and the increment $\Delta I_{v}$, of area $A_{i}$ and duration $t_{i}$, is added at one or the other of the locations $a$ and $b$. But at both locations on each trial a constant "pedestal" is always added. This is an increment $I_{p}$ of area $A_{i}$ and duration $t_{i}$. Thus on any trial the increment at one location will be $I_{p}$ and at the other 


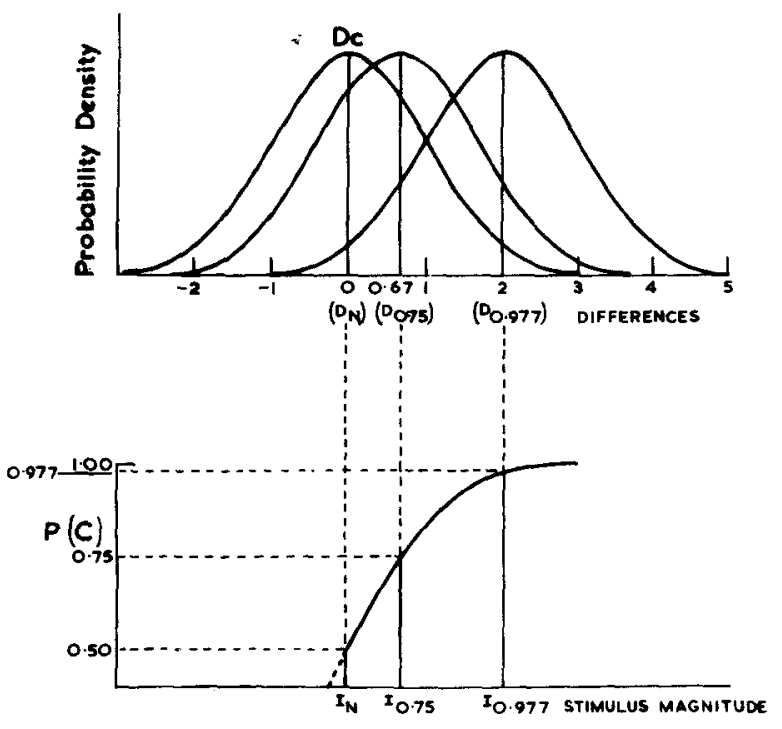

Fig. 2. This illustrates distributions of differences between the central effects of $I_{v}$ and $I_{N}$, when both are presented on a forced-choice trial. (We assume a differencing strategy.) The differences axis is scaled in units of $\sigma_{D}=\sigma_{E_{v}-E_{N}}$, the SD of the distribution of differences, with the origin at $D_{N}=E_{N}-E_{N}=0$, the mean of the distribution of differences given when a zero increment $\Delta I_{v}=0$ is used. The criterion $D_{c}=D_{N}=0 . D_{0.75}=$ $E_{0.75}-E_{N}=D_{N}+0.6745 \sigma_{D}=0.6745 \sigma_{D}$, where $I_{0.75}=$ $I_{N}+\Delta I_{p f c}$ is the stimulus giving $P(C)=0.75$. $D_{0.977}=$ $E_{0.977-} E_{N}=2 \sigma_{D} ; I_{0.977}$ then gives $\mathbf{P}(C)=0.977$. The psychometric function obtained when $P(C)$ is plotted against $I$ is shown in the lower half of the figure.

$\left(\mathrm{I}_{\mathrm{p}}+\Delta \mathrm{I}_{\mathrm{v}}\right)$. The $\mathrm{S}$ must then indicate at which location $\Delta \mathrm{I}_{\mathrm{v}}$ was given.

We shall analyze the three procedures separately.

\section{THE PULSED FORCED-CHOICE PROCEDURE}

It is difficult to apply the model of Fig. 1 to this procedure. When an increment is added to a continuous background as in the cietection procedures, the latter provides a continuing opportunity to take numerous samples that serve to define the $E_{N}$ distribution with little ambiguity. We do not know the extent to which the S's knowledge of the form of the $E_{N}$ distribution is dependent on previous perceptual experience (Treisman, 1964b) and on concurrent sampling of the background during the experimental trials; in either case he needs to know $E_{N}$ to locate $\mathrm{E}_{c}$, the criterion, and the continuous presence of the background will provide this information. But when the stimuli are given as isolated pulses only, the $S$ is not in a position to accumulate and utilize statistical information about the effects of $I_{N}$ in the same way. It seems likely that he will then be forced to use a different procedure for selecting a response, and a common model for this is given below (Tanner \& Swets, 1954).

We suppose that on each trial the $S$ is presented with a pair of stimuli, $I_{N}$ and $I_{v}$, given at locations a and $b$ (the assignment being randomized over trials), and he must report which stimulus is the more intense. The $\mathrm{E}$ records the proportion of "correct" responses, $P(C)$, for each value of $\Delta I_{v}$ that he uses, and takes the threshold for this condition, $\Delta I_{p f}$, as that value of $\Delta I_{v}$ that gives $P(C)=0.75$.

On each trial the $S$ records two central effects, $E_{a}$ and $E_{b}$. He takes the difference between these effects, examines whether it is positive or negative and chooses his response accordingly, i.e., if
$\left(E_{a}-E_{b}\right) \geqslant 0$ he responds " $a$," otherwise " $b$." If $E_{a}$ is in fact $E_{v}$, the effect of $I_{v}$, and he responds " $a$," or if $E_{b}$ is $E_{v}$ and he responds " $b$," the response will be correct. So that $P(C)$ will correspond to the proportion of trials on which the difference $D=E_{v}-E_{N} \geqslant 0$. (If there is bias, i.e., the criterion for selecting between the responses " $a$ " and " $b$ " is not 0 , this will add some extra variance, but as long as the bias is not large we can ignore it.)

Figure 2 illustrates the distribution of $D=E_{\mathbf{v}}-E_{N}$. When $I_{v}=I_{0.75}=I_{N}+\Delta I_{p f c}$, the stimulus giving $P(C)=0.75$, then 0.75 of the area of the distribution of differences will lie to the right of the criterion, $D_{c}=0$. To derive the relation between $\Delta \mathrm{I}_{\mathrm{pfc}}$ and $\mathrm{I}_{\mathrm{N}}$ we need to take account of the variance of this distribution and the relation between $E$ and $I$.

As before, we suppose that $A_{r}=\max \left(A_{i}, \alpha\right)$ and $t_{r}=\max \left(t_{i}, \tau\right)$ but we note that $I_{N}$ is presented only over the area $A_{i}$ and for the duration $t_{i}$, so that

$$
E_{N}=\operatorname{sf}\left(A_{i} t_{i} I_{N}+A_{r} t_{r} I_{n}\right)
$$

and

$$
E_{0.75}=\operatorname{sf}\left[A_{i} t_{i}\left(I_{N}+\Delta I_{p f c}\right)+A_{r} t_{r} I_{n}\right]
$$

Then

$$
E_{0.75}-E_{N}=\operatorname{sfA}_{i} t_{i} \Delta I_{p f c} .
$$

The variance of the $E_{N}$ distribution would be

$$
\begin{aligned}
\sigma_{E(p f c)}^{2}=r_{s} \sigma_{s}^{2} f^{2}\left(A_{i} t_{i} I_{N}\right. & \left.+A_{r} t_{r} I_{n}\right)^{2} \\
& +\left(s^{2}+\sigma_{s}^{2}\right) f\left(A_{i} t_{i} I_{N}+A_{r} t_{r} I_{n}\right)
\end{aligned}
$$

(we will again take $\sigma_{\mathrm{E}}$ as effectively constant over the range of values involved in determining a threshold).

It is evident from Fig. 2 that

$$
E_{0.75}-E_{N}=0.6745 \sigma_{D}=0.6745 \sigma_{E_{0.75}}-E_{N}
$$

From Eqs. 8 and 10 we have

$$
\begin{aligned}
\Delta I_{p f c}= & \frac{0.6745 \sigma_{D}}{s f A_{i} t_{i}} \\
= & \frac{0.6745 \cdot 2^{1 / 2} \cdot\left(1-r_{E}\right)^{1 / 2}}{f A_{i} t_{i}}\left[r_{s} v^{2} f^{2}\left(A_{i} t_{i} I_{N}+A_{r} t_{I} I_{n}\right)^{2}\right. \\
& \left.+\left(1+v^{2}\right) f\left(A_{i} t_{i} I_{N}+A_{r} t_{r} I_{n}\right)\right]^{1 / 2}
\end{aligned}
$$

where $\mathrm{r}_{E}$, the intersample product-moment correlation, must not be confused with $\tau_{s}$, the intrasample correlation. $r_{E}$ is the correlation between the two values of $E$ across trials, and will lie between -1 and +1 . With temporal forced-choice and long interstimulus intervals, IE might be close to 0 . At shorter interstimulus intervals and in spatial forced choice, factors such as spontaneous slow changes in the level of "amplification" of the sensory messages (Wertheimer, 1953) might produce positive values, and effects of the cardiac and respiratory cycles might produce positive or negative values, depending on the length of the ISI.

When $f\left(A_{i} t_{i} I_{N}+A_{r} t_{r} I_{n}\right)$ is small, Eq. 11 will reduce to 


$$
\Delta I_{p f c}=\frac{0.9539\left(1-r_{E}\right)^{3 / 2}}{f^{3 / 2} A_{i} t_{i}}\left(A_{i} t_{j} I_{N}+A_{1} t_{1} I_{n}\right)^{3 / 2}
$$

and when it is large the equation will reduce to

$$
\Delta I_{p f c}=\frac{0.9539\left(1-r_{E}\right)^{1 / 2}}{A_{i} t_{i}} r_{S}^{1 / 3} V\left(A_{i} t_{i} I_{N}+A_{r} t_{r} I_{n}\right)
$$

We can now derive predictions for the effects of the stimulus parameters when the pulsed forced-choice procedure is employed. We consider variation in each parameter, with the others held constant.

Variation in $t_{i}$. This will have different effects, depending on the magnitude of $I_{N}$. (a) Consider $I_{N}$ large, so that Eq. 13 applies and $A_{r} t_{r} I_{n}$ is negligible as compared with $A_{i} t_{i} I_{N}$. Then Eq. 13 reduces to

$$
\Delta \mathrm{I}_{\mathrm{pfc}}=0.9539\left(1-\mathrm{I}_{\mathrm{E}}\right)^{1 / 2} \mathrm{r}_{\mathrm{s}}^{1 / 2} \mathrm{vI}_{\mathrm{N}}
$$

which implies that the threshold is constant and independent of $t_{i}$ however small this is: Bloch's law will not be shown even at stimulus durations well below the critical duration, $\tau$ (as determined by a classical procedure). If $\log \Delta \mathrm{I}_{\mathrm{pfc}}$ is plotted against $\log t_{i}$ this will give a straight line with zero slope. (b) Let $f\left(A_{i} t_{i} I_{N}+A_{r} t_{r} I_{n}\right)$ be small and $A_{r} t_{r} I_{n}$ negligible as compared with $A_{i} t_{j} I_{N}$. Then Eq. 12 will apply, and it can be written as $\Delta I_{\mathrm{pfc}}=\mathrm{k} / \mathrm{t}^{1 / 2}$, where $\mathrm{k}$ is constant, for the whole range of values of $t_{i}$. Thus as $I_{N}$ decreases the zero slope given by Eq. 14 (when $\log \Delta I_{p f c}$ is plotted against $\left.\log t_{i}\right)$ will change to a slope of -0.5 , and this will apply for values of $t_{i}$ both above and below $\tau$. Again, Bloch's law is not shown. (c) When $I_{N}$ is small enough for $A_{i} t_{i} I_{N}$ to be negligible as compared with $A_{r} t_{r} I_{n}$, i.e., at or near the absolute threshold, Eq. 12 implies that $\Delta \mathrm{I}_{\mathrm{pfc}}$ will be inversely related to $t_{i}^{3 / 2}$ when $t_{i} \geqslant \tau$, and Bloch's law, a slope of -1 on the log-log plot, will be shown when $t_{i}<\tau$.

Figure 3 shows data obtained by Leshowitz, Taub, and Raab (1968), using the two-alternative temporal forced-choice procedure for a number of values of $I_{N}$ (the parameters of the curves give $\log I_{N}$, in $\log \mathrm{mL}$ ). The open circles give values of the pulsed forced-choice threshold. We see that the temporal summation curves for $\log \mathrm{I}_{\mathrm{N}}=2.3 \log \mathrm{mL}$ and $1.3 \log \mathrm{mL}$ are flat or almost flat over a range of values of $t_{i}$ from 0.07 msec to $320 \mathrm{msec}$. As the background intensity is reduced there is a tendency for the slope of the curves to get steeper, the curves appearing similar both above and below $\tau$ (which here is in the region of $32 \mathrm{msec}$ when the background intensity is $1.3 \log \mathrm{mL}$ ). The approximate overall slopes as $\log \mathrm{I}_{\mathrm{N}}$ decreases from 2.3 to $\overline{2} .3$ are: $0,-0.04,-0.14,-0.25$, and -0.25 . These results are in accordance with predictions (a) and (b) above: both above and below $\tau$ there is a zero slope for $I_{N}$ high, with a change to a negative slope as $I_{N}$ is decreased. Presumably a slope of -0.5 is not shown because a sufficiently low value of $I_{N}$ has not been used. [In the computer simulation study it was found that the slope of partial summation curves was affected by $n$, the exponent of the transducer function $E=1^{n}$, when this was taken to be a power function (Treisman, 1966). We have here taken $n$ to be 1 ; it remains to be seen whether with other acceptable values of $n$ the terminal slope as $I_{N}$ is decreased (when the conditions for prediction (b) apply) may not be greater or less than -0.51 .

Variation in $A_{i}$. The predictions obtained are similar to those for variation in $t_{i}$. As yet these have not been experimentally tested.

Variation in $I_{N}$. Inspection of Eqs. 12 and 13 suggests that the

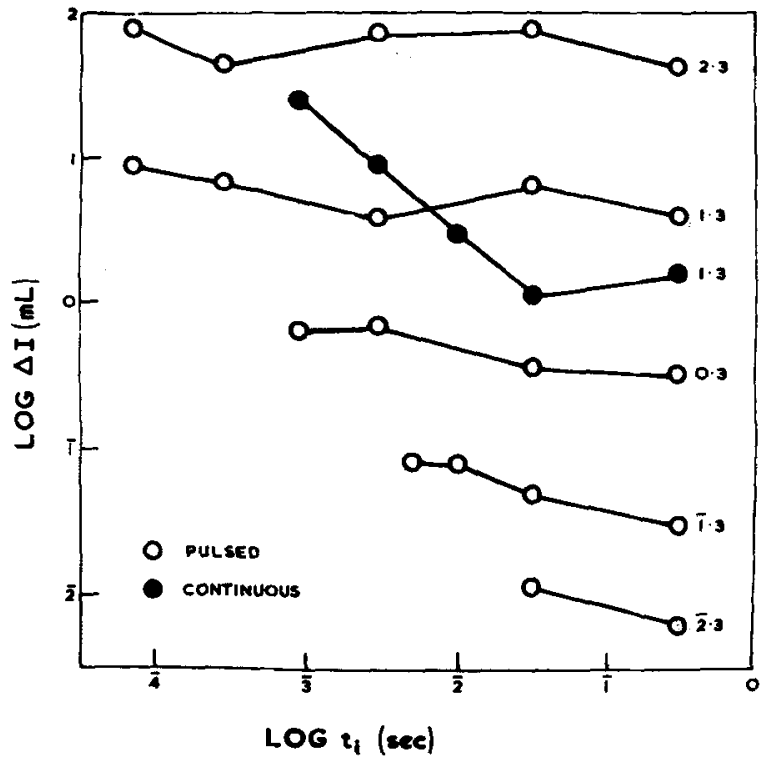

Fig. 3. The relation between the forced-choice threshold and the duration of the increment: data obtained by Leshowitz, Taub, and Raab (1968) by a temporal forced-choice procedure. Each trial began with a warning click; two additional clicks spaced $0.8 \mathrm{sec}$ apart occurred $0.8 \mathrm{sec}$ after the start of the trial and accompanied the two flashes. $O$ : pulsed forced-choice procedure; - continuous forced-choice procedure. The parameters of the curves are the intensities of $I_{N}$ in $\log \mathrm{mL}$. The results of two Os were averaged. The threshold is the value of the increment giving $P(C)=0.75$.

Weber function will show a square root section at low values of $I_{N}$, and become linear at high values, as in Eqs. 4 and 5. However, any prediction about the form of the Weber function is subject to two further considerations. First it should be noted that predictions derived from Eq. 11 will depend on the extent to which $\mathrm{r}_{\mathrm{E}}$ is constant, and should take into account factors that might cause $r_{E}$ to vary. One such factor would be the length of the interstimulus interval. It is conceivable that $\mathrm{r}_{\mathrm{E}}$ will decrease as ISI is increased, being maximal in the spatial forced-choice case and falling to 0 at long interstimulus intervals, and it will be of interest to examine this experimentally. It is also conceivable that $r_{E}$ might decrease as $I_{N}$ increases. Barlow, FitzHugh and Kuffler (1957) noted that changes in the neural organization of the cat's retina occur during the process of dark adaptation, the area of the receptive field increasing, and the inhibitory periphery disappearing when the eye has adapted sufficiently to the dark. If at the higher values of $I_{N}$ the receptive fields are more tightly organized and lateral inhibitory effects more marked, this might tend to reduce the similarity of response of neighboring areas of retina and so reduce $\mathrm{r}_{\mathrm{E}}$. If this effect occurs to a significant extent then as $I_{N}$ is increased $\mathrm{I}_{\mathrm{E}}$ will decline, and consequently $\Delta \mathrm{I}_{\mathrm{p} f \mathrm{c}}$ will increase as a function of $I_{N}$ more rapidly than Eq. 11 suggests.

A second factor that must be considered is the effect of light adaptation, or its absence. In Sections I and II, the statistical decision model was applied to procedures concerned with sensitivity to small departures from an intensity level to which the eye is adapted. The transducer function, $E=f(I)$, was assumed to describe the response of the visual pathway at and near such an adaptation level, and on this assumption was found to be a power function, $E=I^{n}$, with $n$ ranging about 1.0. Consequently we have developed the model for the case where $n=1$. But in the pulsed FCP, $I_{N}$ and $\left(I_{N}+\Delta I_{v}\right)$ are both flashed 
onto a dark-adapted retina, not a retina adapted to $I_{N}$; thus, especially when we are considering variation in $I_{N}$, the effects of the level of adaptation cannot be ignored. At low levels of $I_{N}$ the eye will be more or less appropriately adapted; at high levels it will be inadequately adapted, so that the volume of messages transmitted along the visual pathways will be excessive, and the fibers involved will be increasingly forced towards their upper limiting response rates. This should make them less responsive to further stimulation, so that the greater $I_{N}$ is, the less the response to a further increase in stimulus intensity will be. This negatively accelerated response to increase in I corresponds to a transducer function with $\mathbf{n}$ less than one. Thus we might expect an increase in $I_{N}$ effectively to reduce the value of $n$ in the transducer function, $E=I^{n}$. In a computer simulation of the application of the statistical decision model in which the effects of varying the parameters were studied, it was found that either decrease in the level of adaptation, or decrease in the exponent of the transducer function would cause a rise in threshold, the rise being greater when the two factors are combined (see Figs. 5 and 6 in Treisman, 1966). As $I_{N}$ is increased in the pulsed FCP the level of adaptation will be increasingly inadequate, and the exponent of the transducer function may fall too; thus $\Delta \mathrm{I}_{\mathrm{pfc}}$ should rise more rapidly as $I_{N}$ is increased than is suggested by Eq. 11 since the latter assumes adequate adaptation and a constant transducer function.

Thus both the possible decline in $\mathrm{T}_{\mathrm{E}}$ as $\mathrm{I}_{\mathbf{N}}$ increases, and the absence of adaptation to $I_{N}$, suggest that the Weber function will be steeper than indicated by Eq. 11 . Some data bearing on this prediction will be discussed after the continuous FCP has been considered.

\section{THE CONTINUOUS FORCED-CHOICE PROCEDURE}

In a second variant of the FCP, the "continuous forced-choice procedure," $I_{N}$ is a constant background that is continuously present, not restricted in its presentation to the same area and duration as the increment, and $\Delta \mathrm{I}_{\mathrm{v}}$ is added to one or the other of two spatial or temporal locations. We shall assume in considering this procedure that the two possible sites or times of occurrence of the increment are sufficiently clearly indicated to the $S$ for any uncertainty about the times or positions of occurrence of the stimuli to be negligible (Treisman, 1964b).

In relation to this procedure we shall consider two main strategies that the $S$ might adopt:

\section{The Differencing Strategy}

On each trial the $S$ may record two inputs, $E_{a}$ from location a and $E_{b}$ from location $b$, and use the difference between these inputs to determine his response. Thus if $\left(E_{a}-E_{b}\right) \geqslant 0$ he may respond " $a$," otherwise " $b$." If $E_{a}$ is in fact $E_{v}$, the effect of $I_{v}$, and he responds " $a$," or if $E_{b}$ is $E_{v}$ and he responds " $b$," the response will be correct. Thus $P(C)$ will correspond to the proportion of trials on which the difference $D=E_{\mathbf{v}}-E_{N} \geqslant 0$ (see Fig. 2). This is the identical strategy that was assumed in developing a model for the pulsed forced-choice procedure, and we derive predictions from it in the same way. As before, we let $A_{r}=\max \left(\hat{A}_{i}, \alpha\right)$ and $t_{r}=\max \left(t_{i}, \tau\right) ; \Delta I_{c f} c^{\prime}$ is the continuous forced-choice threshold given when the differencing strategy is applied. When $I_{V}=I_{0.75}=I_{N}+\Delta I_{c f} c^{\prime}$ then $P(C)=0.75$ and 0.75 of the area of the distribution of differences, $D=E_{v}-E_{N}$, will lie to the right of the criterion $D_{c}=E_{N}-E_{N}=0$. Then we have

$$
E_{N}=\operatorname{sfA}_{r} t_{r}\left(I_{N}+I_{n}\right)
$$

and

$$
E_{0.75}=\operatorname{sf}\left[A_{r} t_{r}\left(I_{N}+I_{n}\right)+A_{i} t_{i} \Delta I_{c f} c^{l}\right]
$$

Then

$$
\mathrm{E}_{0.75}-\mathrm{E}_{\mathrm{N}}=\operatorname{sfA}_{\mathrm{i}} \mathrm{t}_{\mathrm{i}} \Delta \mathrm{I}_{\mathrm{cfc}^{\prime}}
$$

The variance of the $E_{N}$ distribution will be

$$
\sigma_{E}^{2}=r_{s} \sigma_{s}^{2} f^{2} A_{r}^{2} t_{r}^{2}\left(I_{N}+I_{n}\right)^{2}+\left(s^{2}+\sigma_{S}^{2}\right) f A_{r} t_{T}\left(I_{N}+I_{n}\right) \text {. }
$$

We see from Fig. 2 that $E_{0.75}-E_{N}=0.6745 \sigma_{D}=$ $0.6745 \sigma_{E_{0.75}-F_{N}}$. Then

$$
\begin{aligned}
\Delta I_{c f c^{\prime}}= & 0.6745 \sigma_{D} / s f A_{i} t_{i} \\
= & \frac{0.6745 \cdot 2^{1 / 2}\left(1-r_{E}\right)^{3 / 2}}{f A_{i} t_{i}}\left[r_{s} v^{2} f^{2} A_{r}^{2} t_{r}^{2}\left(I_{N}+I_{n}\right)^{2}\right. \\
& \left.+\left(1+v^{2}\right) f A_{r} t_{r}\left(I_{N}+I_{n}\right)\right]^{1 / 2} .
\end{aligned}
$$

When $f_{\mathbf{r}} t_{r}\left(I_{N}+I_{n}\right)$ is small this reduces to

$$
\Delta I_{c f c^{\prime}}=\frac{0.9539\left(1-r_{E}\right)^{1 / 2}}{f^{1 / 2} A_{i} t_{i}} A_{I}^{1 / 2} t_{r}^{1 / 2}\left(I_{N}+I_{n}\right)^{1 / 2}
$$

and when this is large Eq. 19 reduces to

$$
\Delta I_{c f c^{\prime}}=\frac{0.9539\left(1-r_{E}\right)^{1 / 2}}{A_{i} t_{i}} r_{s}^{1 / 2} v A_{r} t_{r}\left(I_{N}+I_{n}\right)
$$

It is evident that Eq. 19 resembles Eq. 3 with $\mathrm{z}$ replaced by $0.9539\left(1-r_{E}\right)^{1 / 2}$, and it leads to similar predictions for spatial and temporal summation.

Variation in $t_{i}$. (Since $\mathrm{I}_{\mathrm{N}}$ and ISI are both constant, $\mathrm{r}_{\mathrm{E}}$ may be taken to be constant.) When $t_{i}>\tau$, variation in $t_{i}$ will give $\Delta \mathrm{I}_{\mathrm{cfc}} \mathrm{c}^{\prime}=\mathrm{k}$ when Eq. 21 holds and $\Delta \mathrm{I}_{\mathrm{cfc}}=\mathrm{k}^{\prime} / \mathrm{t}_{\mathrm{i}}^{1 / 2}$ when Eq. 20 holds. For $\mathrm{t}_{\mathrm{i}} \leqslant \tau$, Bloch's law is given in each case.

Variation in $A_{i}$. Similar predictions are given.

Variation in $I_{N}$. The presence of the term $\left(1-\mathrm{r}_{\mathrm{E}}\right)^{1 / 2}$ in Eq. 19 will have the same implications as its presence in Eq. 11. Thus if variation in $r_{E}$ affects the form of the pulsed FCP Weber function, it will have the same effect on the continuous FCP Weber function, assuming that the differencing strategy is employed in the latter procedure. But any effect of inadequate adaptation to $I_{N}$ on the pulsed FCP Weber function will not be shown for the continuous procedure, since in the latter $I_{N}$ is constantly present. This will be discussed further below.

We see that if in the continuous forced-choice procedure the $S$ applies the differencing strategy, we should expect the effects of the increment duration and area on the threshold to resemble those found when the detection procedures are employed, rather than those that result from applying the differencing strategy in the pulsed FCP. The continuous FCP data in Fig. 3 are in agreement with the prediction for temporal summation: a curve is shown that gives values of $\Delta I_{c f c}$ determined against a constant background of $1.3 \log \mathrm{mL}$. In this case the data approximate closely to Bloch's law when $t_{i}$ is below a value in the region of $32 \mathrm{msec}$ (the slope on the log-log plot is approximately -0.9) and the curve flattens out above this value. The curve is notably different from those given with the pulsed FCP.

\section{The Double Detection Strategy}

It has commonly been assumed that the differencing strategy is always applied in the FCP, although, in fact, other strategies are 
possible. Thus Tanner and Swets (1954) assume what is essentially a differencing strategy for a visual intensity discrimination using the continuous forced-choice procedure. However, Blackwell (1963) argued that their data were not fully consistent with this assumption and proposed instead a "high threshold" model similar to the alternative strategy that will be described below.

The "double detection strategy" is the separate application, to each of the two locations that the $S$ must choose between, of the strategy for the detection procedures that was described in Section II and illustrated in Fig. 1. As the background is continuously present, the $S$ is in the position of having a "noise" distribution to which each input sample can be related, as with the detection procedures. Since on each trial there are two locations at which the increment might occur, the response could be determined in two stages. Thus on a given trial the discriminatory mechanisms might compare the input $\mathrm{E}_{\mathrm{a}}$ with a criterion $E_{c(a)}$ (corresponding to $E_{c}$ in Fig. 1), and if $E_{a} \geqslant E_{c(a)}$ make and store a "covert positive judgment." The input $\mathrm{E}_{\mathrm{b}}$ might be less than the corresponding cirterion, $E_{c}(b)$, giving rise to a convert negative judgment, and the $S$ would then proceed to make an overt response indicating that the increment had occurred at location a. If a covert detection occurred at both a and $b$, or no detection occurred at either location, we might suppose that the $S$ would then respond at random.

Obviously, other strategies could also be suggested. For example, the $S$ might apply a hybrid procedure in which he employs the double detection strategy, but when covert detections occur both at $a$ and $b$, or at neither $a$ nor $b$, he reverts to considering the sign of the difference $\left(E_{a}-E_{b}\right)$. However, we shall here limit our consideration to the double detection strategy as described above.

This strategy may seem less elegant than the differencing procedure, but under some experimental conditions its application might have advantages. If very long ISIs are used in temporal forced choice, the possibility of decay in memory would become important. With the double detection strategy the information to be retained from the first stimulus presentation on each trial is not more than one bit-whether the first covert judgment was positive or negative. With the differencing strategy, the actual value of the first input, $E_{a}$, must be retained in memory, and this may constitute a considerably greater load. Experiments in which an accessory stimulus followed a critical stimulus increment at various intervals, and the ability of the accessory stimulus to affect the criterion, $E_{\mathfrak{c}}$, used in detecting the critical increment was studied, have provided evidence that sensory inputs can be retained in memory in uncategorized form for periods only up to $0.5-1.0 \mathrm{sec}$ (Treisman, 1964b). This makes it seem unlikely that differencing could be applied at long ISIs. If double detection is applied at long interstimulus intervals, then as the ISI decreases there might be a transition to the differencing strategy, but it is not obvious that this must occur. Since it is likely that in daily life the detection of isolated stimulus increments is a far more common and important necessity for the senses than the making of forced choices, it is quite possible that there may be a strong bias for the sensory mechanisms to operate in the detection mode whenever the presence of a continuous background makes this possible.

If the double detection strategy is applied, two stimuli, $I_{N}$ and $I_{v}=I_{N}+\Delta I_{v}$, being presented on each trial, then the probability of a "correct" response on any trial, $P(C)$ will depend on $P\left(P J \mid I_{v}\right)$, the probability of a positive covert judgment for the location at which $I_{v}$ is presented, which corresponds exactly to $P\left(Y \mid I_{v}\right)$ for the method of constant stimuli, and on $P\left(P J \mid I_{N}\right)$ the probability of a positive judgment at the location that does not have the increment, which corresponds to the false positive rate $F P R=P\left(Y \mid I_{N}\right)=\&$. For a given value of $I_{v}$

$$
\begin{aligned}
& P(C)=P\left(P J \mid I_{v}\right)\left[1-P\left(P J \mid I_{N}\right)\right] \\
& +1 / 2\left\{P\left(P J \mid I_{v}\right) P\left(P J \mid I_{N}\right)+\left[1-P\left(P J \mid I_{v}\right)\right]\left[1-P\left(P J \mid I_{N}\right)\right]\right\} \\
& =0.5\left[\mathbf{P}\left(\mathbf{P J} \mid \mathbf{I}_{\mathbf{v}}\right)+1-\varepsilon\right] \text {. }
\end{aligned}
$$

The value of $I_{y}$ that gives $P(C)=0.75$ is often used to define the continuous forced-choice threshold, $\Delta \mathrm{I}_{\mathrm{cfc}}$. (We shall use $\Delta I_{\text {cfc }}$ " to represent the continuous forced-choice threshold given when the double detection strategy is applied.) In a trained $S$ who maintains his false positive rate, \&, at a low level, it will be approximately true that the value of $I_{y}$ that gives $P(C)=0.75$ will be the value that gives $P(P J)=0.5$; i.e., it will be the value represented as $I_{0.50}$ in Fig. 1, which gives the threshold $\Delta \mathrm{I}=\mathrm{I}_{0.5}-\mathrm{I}_{\mathrm{N}}$ when the method of constant stimuli is used. Thus if the $S$ employs the double detection strategy, then $\Delta \mathrm{I}_{\mathrm{cfc}}$ " will be a good approximation to $\Delta \mathrm{I}$ as obtained by the detection procedures, and so Eq. 3 will predict all the effects of stimulus parameters on $\Delta \mathrm{l}_{\mathrm{cfc}}$ "; i.e., Bloch's law will hold below $\tau$, and, Ricco's law will hold below $\alpha$, and the Weber function will be the same as that given by the detection procedures.

We can now compare the claims of the differencing and double detection strategies as possible descriptions of the decision processes underlying the determination of the continuous forced-choice threshold. We have seen that both strategies predict the same spatial and temporal summation curves as does the model applied to the detection procedures in Section II, and that, for temporal summation, this accords with the data for $\Delta I_{c f c}$ shown in Fig. 3. Thus either strategy would be adequate to explain the effects of $A_{i}$ or $t_{i}$ on $\Delta I_{c f c}$. However, if $I_{N}$ is varied, the predictions given by the two strategies may differ.

Variation in $I_{N}$. The Weber function for the detection procedures, which derives from Eq. 3, has been described. The Weber function for the pulsed FCP is given by Eq. 11. It contains the term $\left(1-\tau_{E}\right)^{1 / 2}$. We have suggested that $\tau_{E}$ might decrease as $I_{N}$ increases, and we have noted that in the pulsed FCP the retina is not adapted to $I_{N}$; either or both factors would cause $\Delta I_{p f c}$ to rise more rapidly as $\mathrm{I}_{\mathbf{N}}$ increases than is the case in the detection procedures. It is of interest, then, to see whether the pulsed FCP Weber function is in fact steeper than that obtained from the detection procedures. If it is, this would support our analysis of the pulsed FCP, and it would then be of interest to examine the form of the Weber function for the continuous FCP. The expression for $\Delta \mathrm{I}_{\mathrm{cfc}^{\prime}}, \mathrm{Eq} .19$, contains the term $\left(1-\mathrm{r}_{\mathrm{E}}\right)^{1 / 2}$, but the expression for $\Delta \mathrm{I}_{\mathrm{cfc}}$ " does not. So if the $\mathrm{S}$ applies the double detection strategy, the Weber function that results will be similar to that for the detection procedures. But if he applies the differencing strategy, and $\mathrm{r}_{\mathrm{E}}$ decreases to a significant extent as $I_{N}$ increases, he will give a steepened Weber function.

Figure 4 shows Weber functions obtained using the pulsed and continuous forced-choice procedures by Cornsweet and Pinsker (1965), and Leshowitz, Taub, and Raab (1968). Cornsweet and Pinsker's data were obtained using spatial forced choice with $t_{i}=4.5 \mathrm{msec}$ for pulsed forced choice and $3.6 \mathrm{msec}$ for continuous forced choice [the data shown in Fig. 4 are for S DO; results for two pulsed FCP experiments (I and III) have been averaged together]. To find the threshold Cornsweet and Pinsker used a staircase procedure. Their rule for varying the stimulus was: after one wrong response increase $I_{v}$; decrease $I_{v}$ only after two correct responses. This procedure estimates the $71 \%$ point (Wetherill \& Levitt, 1965), thus both $\Delta \mathrm{I}_{\mathrm{pfc}}$ and $\Delta \mathrm{I}_{\mathrm{cfc}}$ are defined by $P(C)=0.71$ for their results. Leshowitz, Taub, and Raab (1968) used temporal forced choice; the data in Fig. 4 (the results of two Ss averaged together) were obtained with $t_{i}=32 \mathrm{msec}$. Each trial began with a warning click. Two additional clicks spaced $0.8 \mathrm{sec}$ apart occurred $0.8 \mathrm{sec}$ after the start of the trial and accompanied the two flashes. $P(C)$ was determined for a 


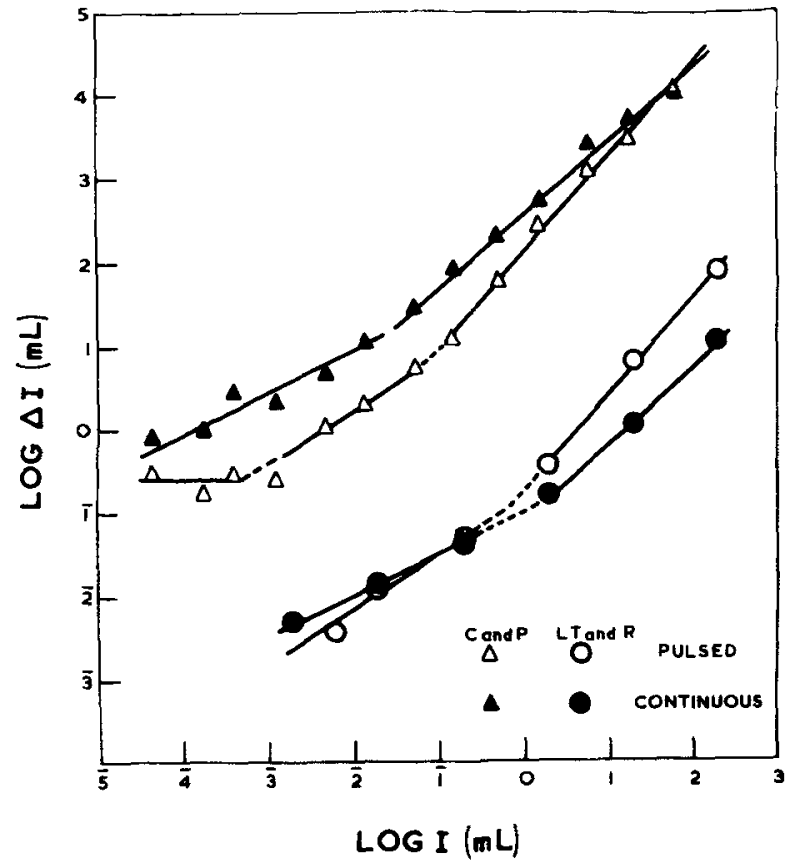

Fig. 4. Log $\Delta I$ plotted against $\log I$ (both in $\log \mathrm{mL}$ ): pulsed and continuous forced-choice thresholds obtained by Cornsweet \& Pinsker (1965) [spatial forced choice: S DO, the results for two pulsed forced-choice experiments (I and III) have been averaged together] and Leshowitz, Taub, and Raab (1968) (temporal forced choice, the results for two Ss have been averaged together). In the latter case the threshold is the value of the increment giving $P(C)=0.75$. The data obtained by Cornsweet and Pinsker have been shifted upwards $3 \mathrm{log}$ units. The straight lines, fitted by eye, have the following slopes (from left to right): for the continuous forced-choice data-0.5 and 0.9 ; for the pulsed forced-choice thresholds-0 (for one curve), 0.67 , and 1.15 .

number of values of $I_{V}$ and the threshold, the value of $\Delta I_{v}$ giving $P(C)=0.75$, was obtained from the resulting psychometric function.

Straight lines have been fitted by eye to the Weber functions. It can be seen that, to a first approximation, both sets of continuous forced-choice data can be described as consisting of two segments, the first with a slope of 0.5 on the $\log -\log$ plot, the second with a slope of 0.9 . Cornsweet and Pinsker's pulsed forced-choice data show a segment at absolute threshold (zero slope); beyond this both sets of $\Delta \mathrm{I}_{\mathrm{pfc}}$ values can be fitted with slopes of 0.67 (when $I_{N}$ is low) increasing to 1.15 (for $I_{N}$ high).

It was predicted that the pulsed FCP Weber function would be steeper than is conventionally found; and this is borne out by both of the sets of pulsed FCP data in Fig. 4. There is a similar increase in slope with both spatial and temporal forced choice, lines of the same slope fitting reasonably well in both cases. Two mechanisms were discussed above that might give rise to an increase in slope: a decrease in $r_{E}$ with increase in $I_{N}$, and the effect of inadequate adaptation to $I_{N}$. Two features of the data argue against the importance of the first factor: (1) The slope for the pulsed FCP functions is about 1.3 times as great as that for $\Delta I_{c f c}$ at both low values of $I_{N}$ and high; this is a greater increase than could readily be explained by an increase in the term $\left(1-r_{E}\right)^{1 / 2}$ alone. (2) If a decrease in $r_{E}$ made an important contribution to the steepness of the curve, we would expect the slope of the spatial forced-choice curve to be greater than that for temporal forced choice with a long ISI, since the former condition would be optimal for insuring a high initial value of $\mathrm{r}_{\mathrm{E}}$, but when the two flashes are separated by a long interval $\mathrm{r}_{\mathrm{E}}$ might well be low even for small values of $I_{N}$, leaving little scope for increase in the term $\left(1-r_{E}\right)^{3 / 2}$. But the spatial and temporal forced-choice data appear to have identical slopes. These results suggest that variation in $\mathrm{r}_{\mathrm{E}}$ as a function of $\mathrm{I}_{\mathrm{N}}$ is not important and that the steep slopes of the pulsed FCP Weber functions are mainly or wholly attributable to the effect of inadequate light adaptation, as discussed above.

The Comsweet and Pinsker (1965) pulsed FCP data in Fig. 4 are the means of two experiments that gave virtually identical results. In the first the $S$ was initially dark adapted, and the field was dark except when the flashes were given. In the second an adapting field at intensity $I_{N}$ was present for part of each trial: "After the observer fixated the adapting field for $15 \mathrm{sec}$, the field was darkened for $5 \mathrm{sec} . .$. The observer then pressed the key to deliver the flash ..... After a brief dark period ... the cycle was repeated [Cornsweet \& Pinsker, 1965]." Thus in this experiment some photochemical adaptation to $I_{N}$ was maintained, but the rapid neural component of light adaptation was absent at the moment when the flashes were given (Crawford, 1947; Baker, 1963). Thus the absence of this component of light adaptation seems mainly responsible for steepening the Weber function.

Two possible strategies for the continuous FCP have been discussed. We have seen that both predict the conventional findings for spatial and temporal summation, and to the extent that data are available (see Fig. 3) this has been supported. It was also shown that the double detection strategy would lead to the conventional Weber function. The differencing strategy, however, would give a steepened function if an increase in the term $\left(1-r_{E}\right)^{1 / 2}$ occurs as $I_{N}$ gets bigger, but not otherwise. (Inadequate light adaptation would, of course, not be a factor in the continuous procedure.) In fact, Fig. 4 shows that the continuous procedure gives a conventional Weber function. Thus the Ss either employed the double detection strategy, or they employed the differencing strategy, and there is no significant decrease in $r_{E}$ as $I_{N}$ increases. As we have seen, this last would be quite consistent with the pulsed FCP results.

It appears that the statistical decision model can be extended to apply to both pulsed and continuous forced-choice procedures. It predicts or accounts for all the main findings. Two strategies can be suggested for the continuous FCP, and the results we have so far considered are not able to distinguish between them.

A remaining point of interest is the size of the thresholds given by the two procedures. We can see from Fig. 4 that at low values of $I_{N}, \Delta I_{p f c}$ is less than $\Delta I_{c f c}$; this superiority is greater for Cornsweet and Pinsker's data than for those obtained by Leshowitz, Taub, and Raab (1968). As $I_{N}$ increases, $\Delta I_{p f}$ increases more rapidly than $\Delta \mathrm{I}_{\mathrm{cfc}}$ and eventually exceeds it, the crossover occurring earlier in Leshowitz, Taub, and Raab's data. These relations follow fairly simply from the model:

(a) If we assume that the double detection strategy is employed, then $\Delta \mathrm{I}_{\mathrm{cfc}}$ " as given by Eq. 4 (for $\mathrm{I}_{\mathrm{N}}$ small) can be compared with $\Delta I_{p f c}$ as given by Eq. 12. (We shall ignore $I_{n}$, and $A_{r}$ and $A_{i}$; for the two experiments being considered here we can assume $A_{r}=A_{i}$.) Then the former equation differs from the latter in two ways: it contains $z$ (of the order 2-6), corresponding to $0.9539(1-\mathrm{rE})^{1 / 2}$ (maximum possible value 1.35 ); clearly this will tend to make $\Delta I_{c f c}$ " the larger. Equation 4 also contains $t_{s}^{3 / 2} / t_{i}$, corresponding to $1 / t_{1}^{1 / 2}$ in Eq. 12. When $t_{i}=t_{r}$ the two expressions will be identical; when $t_{i}<t_{r}=\tau$, the first expression will be greater than the second, again tending to make $\Delta \mathrm{I}_{\mathrm{cfc}}$ " exceed $\Delta I_{p f c}$. Thus we expect $\Delta I_{c f c}>\Delta I_{p f c}$, as shown for small values of $I_{N}$ in Fig. 4, and we expect the difference to be greater the smaller $t_{i}<\tau$ is. In Cornsweet and Pinsker's experiment, $t_{i}$ is of the order of $4 \mathrm{msec}$, in the Leshowitz, Taub, and Raab data it is 
$32 \mathrm{msec}$; as we would expect from the argument above, $\Delta \mathrm{I}_{\mathrm{cfc}}$ exceeds $\Delta \mathrm{I}_{\mathrm{pfc}}$ by a bigger margin in the first case.

When $I_{N}$ is large we compare Eq. 5 with Eq. 13. As before the former contains $\mathrm{z}$, corresponding to the latter's $0.9539\left(1-\mathrm{r}_{\mathrm{E}}\right)^{1 / 2}$; and $t_{r} / t_{i}$ corresponds to $t_{i} / t_{i}=1 . \Delta I_{c f c}$ will be greater than $\Delta I_{p f c}$, and especially so when $t_{i}<\tau$.

Equations 12 and 13 will make the pulsed threshold always less than the continuous threshold. But they do not include the effect of inadequate light adaptation, which, as discussed above, will make the pulsed Weber function increase more rapidly than these equations suggest, and thus result in the crossing over of the pulsed and continuous functions.

(b) If we assume that the differencing strategy is employed, then, for $I_{N}$ small, $\Delta I_{c f c^{\prime}}$, as given by Eq. 20 , is compared with $\Delta I_{p f c}$, given by $E q .12$. Ignoring $I_{n}$, they differ only in that the former includes $t^{\frac{1}{2}} / t_{i}$ and the latter $1 / t^{1 / 2}$. Similarly, for $I_{N}$ large, Eq. 21 has $t_{r} / t_{i}$ where Eq. 13 has $t_{i} / t_{i}$. It follows that for $t_{i} \geqslant \tau$ the equations will give identical thresholds; taking the effects of adaptation into account $\Delta \mathrm{I}_{\mathrm{pfc}}$ would then be always equal to or greater than $\Delta \mathrm{I}_{\mathrm{cfc}} \mathrm{c}^{\prime}$. For $\mathrm{t}_{\mathrm{i}}<\tau$, the equations will give $\Delta \mathrm{I}_{\mathrm{pfc}}<\Delta \mathrm{I}_{\mathrm{cfc}} \mathrm{c}^{\prime}$, and the greater slope of the pulsed FCP Weber function will reverse this relation when $I_{N}$ is sufficiently large. As $t_{i}$ is less in Cornsweet and Pinsker's data than in those of Leshowitz, Taub, and Raab, the greater excess of $\Delta I_{c f c}$ over $\Delta I_{p f c}$ for low values of $I_{N}$ in the former data is to be expected. In the latter data, $t_{i}=32 \mathrm{msec}$, which is of the order of the value of $\tau$ for the $\Delta \mathrm{I}_{\mathrm{cfc}}$ data in Fig. 3; but the latter were determined at $I_{N}=1.3 \log \mathrm{mL}$, and since $\tau$ tends to increase as $I_{N}$ falls it would be larger at low values of $\mathrm{I}_{\mathrm{N}}$ (Keller, 1941).

\section{Further Comparison of the Double Detection and Differencing Strategies}

We have described two strategies that might be employed in the continuous FCP, but the data discussed above have not enabled us to distinguish between them. It seems worth considering a number of points that might help to indicate which strategy is in fact employed.

(1) From the discussion above of the relative sizes of pulsed and continuous thresholds, we see that if $t_{i}$ is made clearly greater than $\tau$, then with the differencing strategy we will have $\Delta \mathrm{I}_{\mathrm{p} \mathrm{fc}} \geqslant \Delta \mathrm{I}_{\mathrm{cfc}}$ for all values of $\mathrm{I}_{\mathrm{N}}$, but with the double detection strategy $\Delta \mathrm{I}_{\mathrm{pfc}}$ may be less than $\Delta \mathrm{I}_{\mathrm{cfc}}$ at low values of $\mathrm{I}_{\mathrm{N}}$. If the latter were found we would then have evidence that the double detection strategy is employed. However, comparable data for both procedures with $t_{i}$ known to be sufficiently large are not yet available.

(2) The differencing and double detection strategies predict different forms of psychometric function. If we refer to Fig. 2, we see that the psychometric function given by the differencing strategy is a half-ogive; it is the normal distribution function taken from the $50 \%$ to the $100 \%$ points. But the psychometric function will have a different form if the double detection strategy is applied (Blackwell, 1963). We saw from Eq. 22 that when the latter strategy is employed $P(C)$ is a linear function of $P\left(P J \mid I_{v}\right)$. But $P\left(P J \mid I_{v}\right)$ can be assumed to equal $P\left(Y \mid I_{v}\right)$, and a plot of $P(Y)$ against $I$, as in Fig. 1, gives a normal ogive psychometric function. Thus, if the double detection strategy is applied, the psychometric function will be an ogive linearly transposed to lie between the asymptotes $P(C)=0.5(1-\&)$ and $P(C)=0.5(2-\&)$ or, when $\&$ is small enough to be ignored, 0.5 and 1.0. Although it follows that the form of the psychometric function obtained from the continuous FCP should indicate which strategy was employed, in practice it may be difficult to determine the form of the psychometric function with sufficient precision. In Fig. 5 the expected form of the psychometric function for each strategy is shown. In the two lower graphs $P(C)$

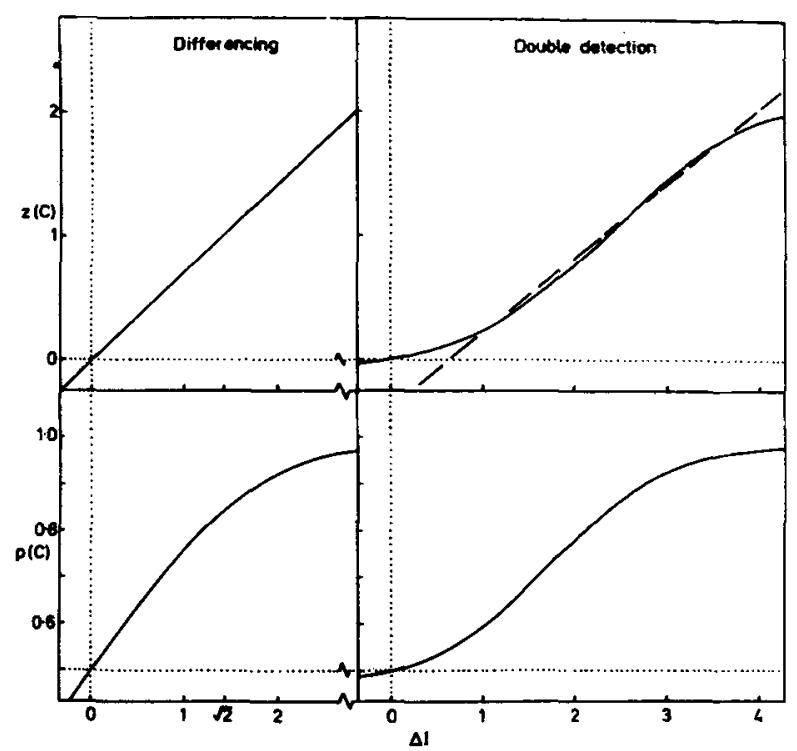

Fig. 5. Theoretical psychometric functions for the differencing and double detection strategies. The lower left curve shows the differencing psychometric function, obtained as in Fig. 2. Above is shown the graph given by plotting the values of $z(C)$, the standardized normal deviates corresponding to values of $P(C)$ below. The lower right curve is the psychometric function for the double detection strategy; values of $P(C)$ are obtained from values of $P(Y)=P(P J)$ (compare Fig. 1) by Eq. 22, assuming $\&=0.04$. The flattened sigmoid curve given when $\mathrm{z}(\mathrm{C})$ is plotted against $\Delta \mathrm{I}$ in this case is shown above. A straight line could be fitted to it reasonably well except at the extremes; the dashed line is an example. For both curves the abscissa is scaled in units of $\sigma_{1}$ (as in Fig. 1); the origin corresponds to $I_{N}$ alone. The SD for the differencing psychometric function, $\sigma_{I(d i f f)}$, is taken to be $2^{1 / 2} \sigma_{1}$.

is plotted against $\Delta \mathrm{I}$. For the double detection strategy, $\mathrm{P}(\mathrm{C})$ is calculated, in accordance with $\mathrm{Eq} .22$, from the values of $P(Y)=P(P J)$ that would be obtained by the method of constant stimuli (as in Fig. 1), assuming that the limiting acceptable false positive rate, $\&=0.04$. The abscissa is plotted in units of $\sigma_{I}$, the SD of the MCS psychometric function. The resulting sigmoid function passes through the point $(0,0.5)$ and its asymptotes are $P(C)=0.48$ and $P(C)=0.98$. The psychometric function for the differencing strategy, derived as in Fig. 2, is a half-ogive; it has been taken that its SD, $\sigma_{I}$ (diff) is equal to $2^{1 / 2} \sigma_{I}$, and the abscissa is marked in units of $\sigma_{I}$. The upper graphs show plots of the corresponding standardized normal deviates, $z(C)$. For the differencing strategy this will be a straight line passing through the origin. For double detection it will be a somewhat flattened sigmoid curve. A dashed straight line, such as might be fitted by probit analysis, is also shown. A fairly good fit to a straight line might be obtained, except for extreme values of the data. The straight line intersects the abscissa at a positive value, not at the origin.

It follows from this that if probit analysis (Finney, 1952) is used to fit continuous forced-choice data, two effects of the strategy chosen may be expected: (a) If the $S$ employs differencing, the straight line should be a good fit, if double detection, a poor fit. (b) For differencing, the straight line should pass through the origin, for double detection it should have a positive intercept with the abscissa.

Figures 6 and 7 show data obtained by Leshowitz, Taub, and Raab (1968) for two Ss. In each case probit analysis has been applied to continuous and pulsed forced-choice data and the 


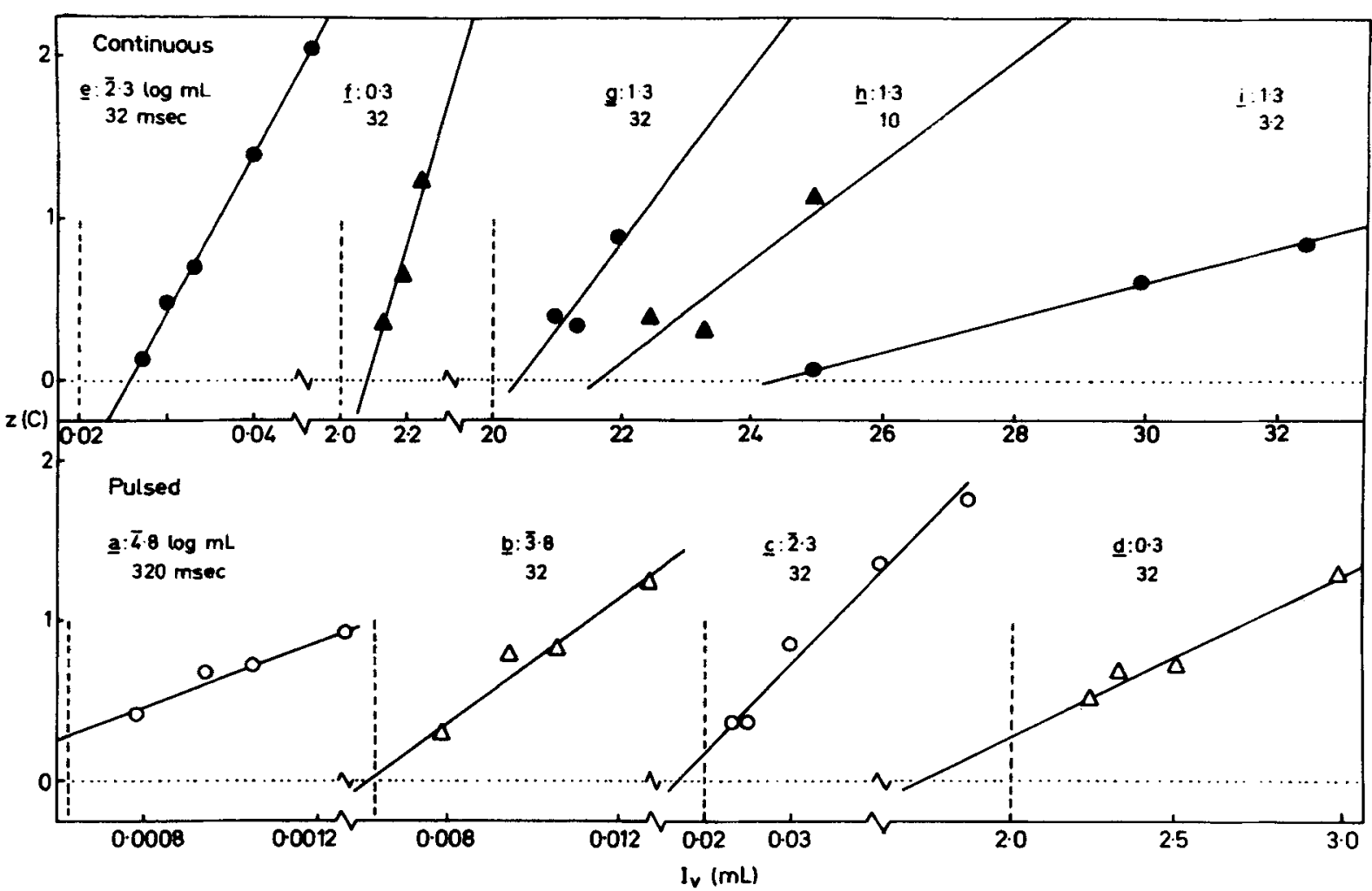

Fig. 6. Data obtained by Leshowitz, Taub, and Raab (1968) for the pulsed and continuous FCPs (S BL). Each value of $P(C)$, the probability of a correct response, has been converted to the corresponding standardized normal deviate, $z(C)$, and a straight line has been fitted to each set of data by probit analysis. The values of $z(C)$ are plotted against $I_{v}=I_{N}+\Delta I_{v}$ (in $\left.m L\right)$. The parameters of the curves are $I_{N}($ in $\log m L)$ and $t_{i}($ in $m s e c)$. The filled circles and triangles represent the continuous FCP data, and the unfilled symbols represent the pulsed FCP data. For each curve the axes $z(C)=0$ and $I_{V}=I_{N}$ are shown as dotted lines.

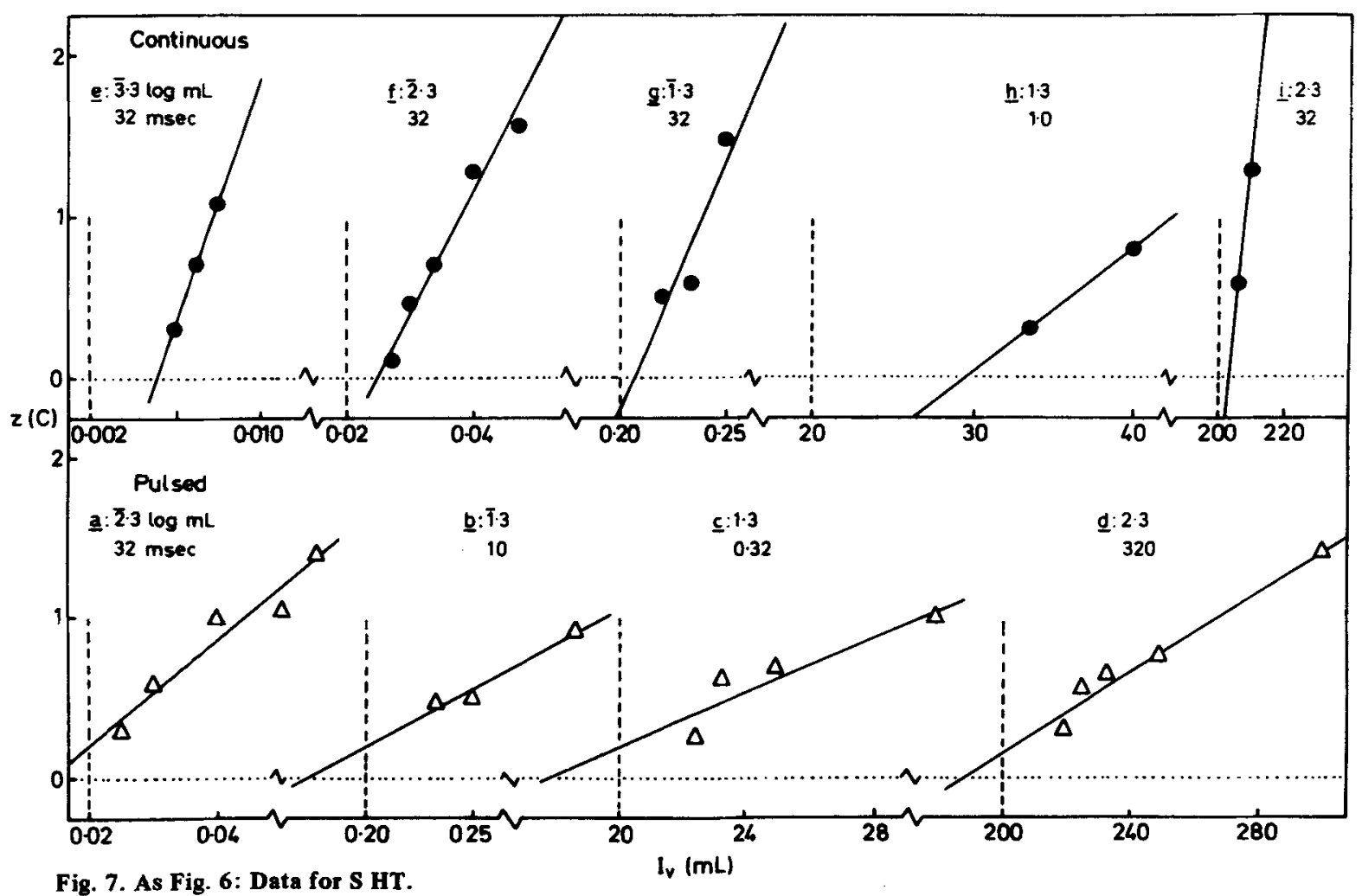




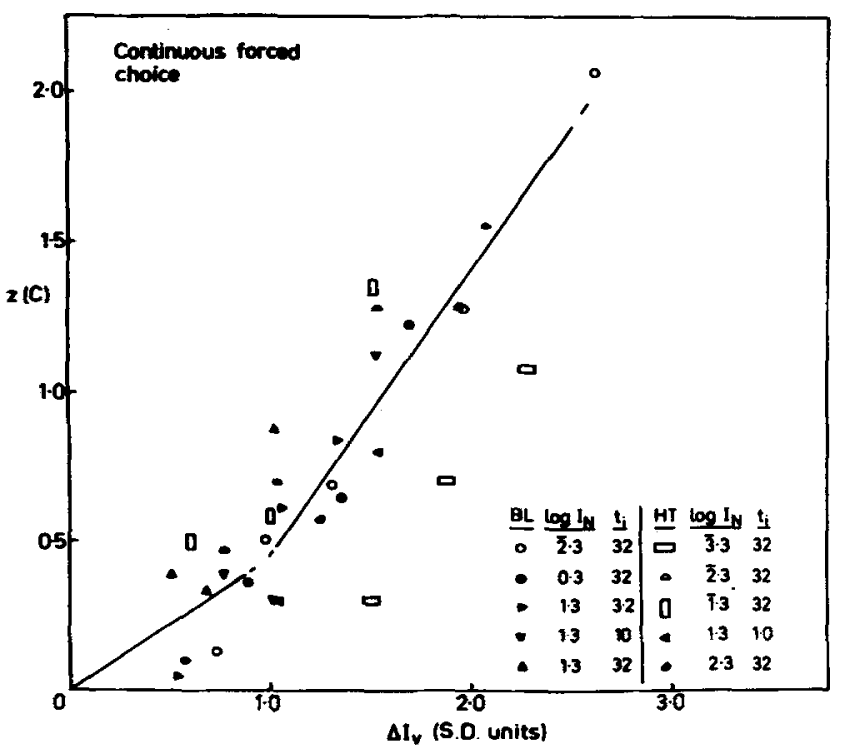

Fig. 8. The continuous forced-choice data shown in Figs. 6 and 7 are shown on a single plot. In each case $\mathbf{z}(C)$ is plotted against $\Delta I_{v}$ standardized by division by the SD given by the probit analysis of the data for that curve. The parameters of the curves, $\log I_{N}(\log m L)$ and $t_{i}(m s e c)$ are shown.

results are presented as plots of $z(C)$ against $I_{V}=I_{N}+\Delta I$, with the best-fitting straight line.

Does probit analysis give a good fit to the continuous FCP data, as differencing would imply, or a poor fit, as we would expect if double detection were the strategy employed? To test this, a value of chi square for goodness of fit was obtained for each line (Finney, 1952), and these values, and their degrees of freedom, were summed for eight curves (the remaining two curves had insufficient data points) to give:

\begin{tabular}{cccl} 
Subject & $\chi^{2}$ & df & Significance \\
\hline BL & 16.10 & 7 & $\mathrm{p}<0.025$ \\
HT & 12.35 & 5 & $\mathrm{p}<0.05$ \\
\hline Both & 28.45 & 12 & $\mathrm{p}<0.005$ \\
\hline
\end{tabular}

This is significant evidence of departure from goodness of fit, which is evidence against the differencing strategy having been employed. It can also be seen that for all 10 curves the intercept on the abscissa is positive; the (one-tailed) probability of this occurring by chance is 1 in $2^{10}$. We saw from Fig. 5 that a deviation in this direction was to be expected if a double detection stretegy were employed. To examine the departure from good fit to the probit line indicated by the high value of chi square, all the continuous FCP curves were replotted in Fig. 8; in each case $z(C)$ was plotted against $\Delta I_{v}$, normalized by division by the SD given by the probit analysis of the given set of data. When the data are put together in this way, the general shape, indicated roughly by the two line segments that were fitted by eye, is compatible with the flattened sigmoid curve passing through the point $(0,0)$, which would be expected if the double detection strategy were applied.

It appears that the results are most consistent with the strategy applied in the continuous FCP being that of double detection rather than differencing, at least in this experiment.

The pulsed forced-choice data obtained in the same experiment are also shown, for comparison. Here the double detection strategy would not be capable of being applied, and we must assume that the Ss will have been obliged to employ the differencing strategy. In this case we would expect the straight lines to pass through the origin, but in all cases the intercept with the abscissa is negative. On the null hypothesis these intercepts would be expected to be equally often positive or negative, so that the probability of a deviation as extreme as this occurring by chance is 1 in $2^{7}$, which is highly significant. This presents a problem whose explanation is not immediately evident.

If the differencing strategy were applied, then, in the absence of further considerations, we would expect the straight lines given by probit analysis to fit well. For these curves the summed value of chi square was $20.33(\mathrm{df}=18)$, which is not significant. However, when nine pulsed FCP curves obtained in a further experiment by Leshowitz, Taub, and Raab (1968) are added in, the overall summed chi square rises to 123.69 ( $\mathrm{df}=44$, $\mathrm{p}<0.001$ ).

To examine whether this high value of chi square resulted from variability or from a consistent departure from the expected shape, all the pulsed FCP curves were plotted in Fig. 9. In each case $z(C)$, the standardized normal deviate corresponding to $P(C)$, was plotted against $\Delta \mathrm{I}_{\mathrm{y}}$, standardized by division by the value of the SD given by the probit analysis for that curve. When the data are plotted in this way it is clear that there is a nonlinear tendency. Because of the randomization involved in the experimental design, we would not expect $P(C)$ for $\Delta I_{v}=0$ to depart significantly from 0.5 [giving $\mathrm{z}(\mathrm{C})=0$ ]. This value of $\Delta \mathrm{I}_{\mathbf{v}}$ was not used, but it is evident that for low values of $\Delta I_{v}$ the data fall along a line passing through or near the point $(0,0)$. However, as $\Delta \mathrm{I}_{\mathrm{V}}$ increases, there is an increasing tendency for the values of $z(C)$ obtained to fall below the level that would be predicted by this initial straight line. This results in a bowing of the curve, roughly illustrated by the two line segments that have been fitted by eye. It is evident that the negative intercepts with the abscissa shown in Figs. 6 and 7 must result from fitting straight lines to this bowed curve.

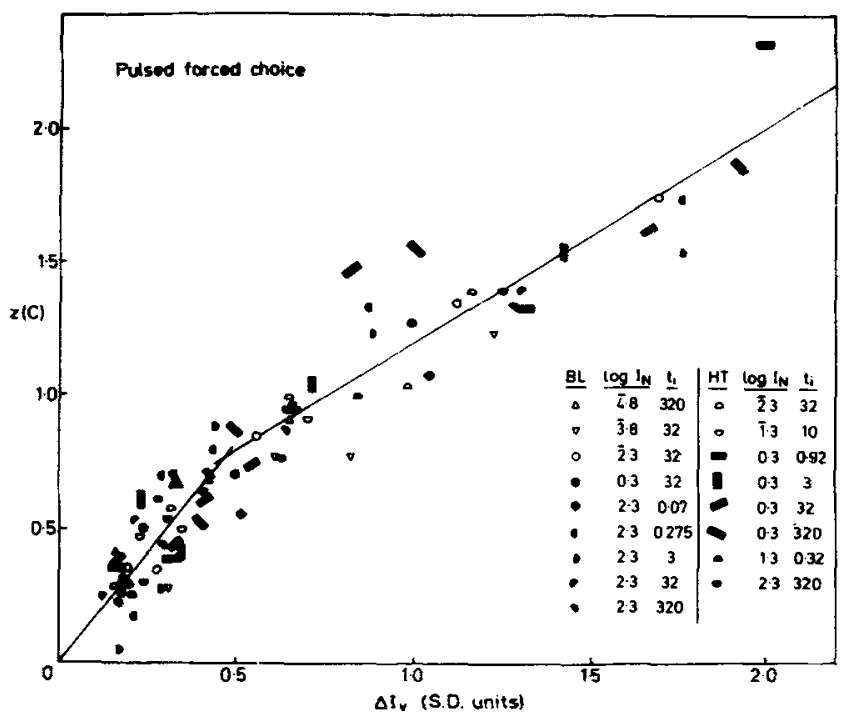

Fig. 9. The pulsed forced-choice data shown in Figs. 6 and 7 , and nine further curves obtained by Leshowitz, Taub, and Raab (1968) are shown on a single plot. $z(C)$ is the standardized normal deviate corresponding to $P(C)$. The values of $\Delta I_{v}$ have been standardized by division by the SD given by the probit analysis of the data for each curve. The parameters of the curves, $\log I_{N}$ in $\log \mathbf{m L}$, and $\mathbf{t}_{\mathbf{i}}$ in $\mathrm{msec}$, are also shown. 
Why does the curve depart from the expected straight line? The most plausible explanation would be that the bowing is an effect of the inadequate light adaptation obtaining in the pulsed forced-choice procedure. Save at the moments when $I_{N}$ or $I_{v}$ are actually being presented, the eye is exposed to an unilluminated background that will be the main determinant of its state of adaptation. The greater $I_{v}$ is, the poorer adaptation to it will be. If poor adaptation effectively increases $\sigma_{\mathbf{E}}(\mathrm{pfc})$, this should produce an unduly poor performance at high values of $I_{v}$. There might also be an effect of the disparity between $I_{v}$ and $I_{N}$. When the first stimulus of the trial is given, this might induce some degree of adaptation to its own level, which might facilitate response to the second stimulus when $\Delta I_{v}$ is small, but impair it when $\Delta \mathrm{I}_{\mathrm{v}}$ is large.

It is instructive to compare Figs. 8 and 9 . As well as the difference in shape in the two cases, the data are more widely scattered in Fig. 8. This would be expected if double detection were applied in the continuous FCP since variation in the criterion $\left(\mathrm{E}_{\mathrm{c}}\right)$ employed for different curves would result in their being laterally dispersed when plotted on the same abscissa. No similar factor would operate to disperse curves given by differencing.

(3) Can information about the strategy employed be derived from the slopes of the functions obtained? If we compare the lines fitted to pulsed and continuous data where they have the same parameters and scales, the latter appear to give steeper slopes. In Fig. 6, curve $\mathrm{e}$ is steeper than $\mathrm{c}$, and $\mathrm{f}$ is steeper than $\mathrm{d}$. In Fig. $7, \mathrm{f}$ is steeper than $\mathrm{a}$; an increase in $t_{i}$ produces a steeper curve (compare curves $g, h$, and $i$, in Fig. 6), yet curve $i$, with $t_{i}=32 \mathrm{msec}$, is steeper than $d$ with $t_{i}=320 \mathrm{msec}$.

If the differencing strategy were applied in the continuous FCP, the slope of the resulting psychometric function would depend on its SD, $\sigma_{\text {Icf }}$. If we refer to Fig. 2 , it is evident that this must depend on the SD of the distribution of differences, $\sigma_{D}=2^{1 / 2}\left(1-r_{E}\right)^{1 / 2} \sigma_{E}$, and the transducer function $E=f(I)$. Making the usual linear assumption for the latter, and taking $\sigma_{\text {Icfe' }}=I_{0.84}-I_{0.50}$, by an argument identical to that embodied in Eqs. 15 to 19 we get

$$
\sigma_{\mathrm{Icfc}}=\sigma_{\mathrm{D}} / \mathrm{sfA}_{\mathrm{i}} t_{\mathrm{i}}=2^{1 / 2}\left(1-\mathrm{r}_{\mathrm{E}}\right)^{1 / 2} \sigma_{\mathrm{E}} / \mathrm{sfA}_{\mathrm{i}} \mathrm{t}_{\mathrm{i}}
$$

If the double detection strategy is applied in the continuous FCP, the resulting psychometric function is simply related to that obtained when such a detection procedure as the method of constant stimuli (MCS) is employed (see Fig. 1). The SD of the latter, $\sigma_{I}$, corresponds to $E_{0.84}-E_{0.50}$, and by an argument parallel to that leading to Eq. 3 we get

$$
\sigma_{1}=I_{0.84}-I_{0.50}=\sigma_{E} / s f A_{i} t_{i}
$$

In Fig. 5 the abscissa is scaled in units of $\sigma_{1}$. If the MCS psychometric function were plotted on this scale, the $z(C)$ transformation would of course be a straight line with a slope of 1. For this figure we have taken $\sigma_{\mathrm{Icfc}}{ }^{\prime}=2^{1 / 2} \sigma_{1}$, and accordingly the $\mathrm{z}(\mathrm{C})$ transform of the differencing psychometric function has a slope of $1 / 2^{3 / 2}=0.71$. The double detection psychometric function is related to the MCS function by Eq. 22; the latter lies between the asymptotes 0 and 1 , the former between $0.5(1-\&)$ and $0.5(2-\&)$ and it is correspondingly flatter. For example, the stimuli that give $P(Y)=0.50$ and $P(Y)=0.84$ when the MCS is applied, will give (ignoring \&) $P(C)=0.75$ and $P(C)=0.92$ for double detection. This would correspond to a slope, on Fig. 5, of 0.73 . With $\&=0.04$, the slope for this portion of the curve would be 0.67 . The dashed approximating straight line shown for the $z(C)$ transform of the double detection curve has a slope of 0.61 .

Evidently, the slope of the probit line is not likely to distinguish between the two strategies. But if both strategies would give probit lines of similar slope, what can we say of the evidence noted above that the continuous data give steeper lines than the pulsed data? Two reasons for this might be suggested: (a) The differencing strategy is employed in both procedures, and the slope for the continuous FCP is determined by $\sigma_{1 \mathrm{cfc}} \mathrm{c}^{\prime}$, as given by Eq. 23. For the pulsed FCP the slope is determined by the SD of the pulsed FCP psychometric function, $\sigma_{1 p f c}$. Referring to Fig. 2 and Eqs. 6 to 11 we see that this is given by

$$
\sigma_{I p f c}=2^{1 / 2}\left(1-T_{E}\right)^{1 / 2} \sigma_{E(p f c)} / s f A_{i} t_{i} .
$$

Then the continuous FCP might give steeper slopes because $\mathrm{rE}_{\mathrm{E}}$ is greater in the continuous procedure than in the pulsed procedure. However, there is no obvious reason why this should be the case, and since the two stimuli on each trial are given at a spacing of $0.8 \mathrm{sec}$, we should not expect $\mathrm{T}_{\mathrm{E}}$ to be large for either procedure. Thus this explanation does not appear plausible. (b) Whether differencing or double detection is employed in the continuous $F C P$, the slope will depend on $\sigma_{E}$ (Eqs. 23 and 24), and the slope in the pulsed FCP will depend on $\sigma_{\mathrm{E} \text { (pfc) }}$ (Eq. 25). Comparing Eq. 9 with Eq. 2 (for the double detection strategy) or Eq. 18 (for differencing) suggests that $\sigma_{\mathrm{E}}(\mathrm{pfc})$ will be less than or equal to $\sigma_{\mathrm{E}}$ for the continuous cases. However, we have noted earlier that in the pulsed FCP adaptation to $I_{N}$ is imperfect, and this would tend to give increased values of $\sigma_{E}(p f c)$ and so a flatter slope for the pulsed FCP. Thus the effect of inadequate light adaptation in the pulsed procedure could explain both the steeper Weber functions and the flatter psychometric functions.

Concluding this section, it appears that our model is capable of predicting the main findings for the continuous forced-choice procedure, as for the others previously considered. We have distinguished two strategies that Ss might employ, and have obtained some evidence that the double detection, rather than the differencing strategy, is the preferred one in the continuous procedure.

\section{THE PEDESTAL FORCED-CHOICE PROCEDURE}

In this procedure a background of intensity $I_{N}$ is constantly present; an increment, $\Delta \mathrm{I}_{\mathrm{v}}$, is given at one or the other of two indicated spatial or temporal locations; at each location an increment $I_{p}$, the "pedestal," is also given, whether or not $\Delta I_{v}$ is added at that location. This procedure is of interest since $I_{N}$ is constantly present and so the state of light adaptation may be better than in the pulsed procedure, especially when $I_{N}$ is large as compared with $I_{p}$. But the $S$ is likely to employ the differencing strategy when $I_{p}$ is at all large, since the double detection strategy would then give an unduly high proportion of detections at both locations on each trial. Thus the use of the differencing strategy may be dissociated from the effects of poor light adaptation.

Making the same assumptions about the sampling and decision mechanisms as before, we obtain

$$
E_{N}=\operatorname{sf}\left[A_{r} t_{r}\left(I_{N}+I_{n}\right)+A_{i} t_{i} I_{p}\right]
$$

and

$$
E_{0.75}=s f\left[A_{r} t_{r}\left(I_{N}+I_{n}\right)+A_{i} t_{i}\left(I_{p}+\Delta I_{p e d}\right)\right],
$$

where $\Delta \mathrm{I}_{\text {ped }}$ is the increment giving $P(C)=0.75$. If the differencing strategy is employed, Fig. 2 will apply, and we will have

$$
E_{0.75}-E_{N}=\operatorname{sfA} A_{i} t_{i} \Delta I_{p e d}=0.6745 \sigma_{D}
$$

Since 


$$
\begin{aligned}
\sigma_{E(\text { ped })}^{2}=r_{s} \sigma_{s}^{2} f^{2}\left[A_{r} t_{r}\left(I_{N}+I_{n}\right)+A_{i} t_{i} I_{p}\right]^{2} \\
+\left(s^{2}+\sigma_{s}^{2}\right) f\left[A_{r} t_{T}\left(I_{N}+I_{n}\right)+A_{i} t_{i} I_{p}\right]
\end{aligned}
$$

we will have

$$
\begin{aligned}
\Delta I_{\text {ped }}= & 0.6745 \cdot 2^{1 / 2}\left(1-r_{E}\right)^{1 / 2} \sigma_{E(p e d)} / s f A_{i} t_{i} \\
= & \frac{0.9539\left(1-r_{E}\right)^{1 / 2}}{f A_{i} t_{i}}\left\{r_{s} v^{2} f^{2}\left[A_{r} t_{r}\left(I_{N}+I_{n}\right)+A_{i} t_{i} I_{p}\right]^{2}\right. \\
& \left.+\left(1+v^{2}\right) f\left[A_{r} t_{T}\left(I_{N}+I_{n}\right)+A_{j} t_{i} I_{p}\right]\right\}^{1 / 2}
\end{aligned}
$$

When $f\left[A_{r} t_{r}\left(I_{N}+I_{n}\right)+A_{i} t_{i} I_{p}\right]$ is small this will reduce to

$$
\Delta I_{\text {ped }}=\frac{0.9539\left(1-r_{E}\right)^{1 / 2}}{f^{1 / 2} A_{i} t_{i}}\left[A_{r} t_{r}\left(I_{N}+I_{n}\right)+A_{i} t_{i} I_{p}\right]^{1 / 2}
$$

and when it is large to

$$
\Delta I_{\text {ped }}=\frac{0.9539\left(1-r_{E}\right)^{1 / 2}}{A_{i} t_{i}} r_{s}^{1 / 2} v\left[A_{r} t_{r}\left(I_{N}+I_{n}\right)+A_{i} t_{i} I_{p}\right]
$$

Also

$$
\sigma_{\text {Iped }}=I_{0.84}-I_{0.50}=\frac{2^{1 / 2}\left(1-r_{E}\right)^{1 / 2} \sigma_{E}(\text { ped })}{s f A_{i} t_{i}} .
$$

We can now derive predictions for this procedure.

Variation in $t_{i}$. Consider $I_{N}$ large, so that Eq. 32 applies and $I_{n}$ is negligible. Then for $t_{i} \geqslant \tau, \Delta I_{\text {ped }}$ is constant. For $t_{i}<\tau$, the equation can be simplified to $\Delta \mathrm{I}_{\text {ped }}=\mathrm{k} / \mathrm{t}_{\mathrm{i}}+\mathrm{k}^{\prime}$, with $\mathrm{k} \propto \mathrm{I}_{\mathrm{N}}$ and $\mathbf{k}^{\prime} \propto \mathbf{I}_{\mathbf{p}}$. The shape of the temporal summation curve will then depend on the relative magnitudes of $k$ and $k^{\prime}$. For $I_{p}=0$ (the continuous procedure) we would have Bloch's law, with a slope of -1 when $\log \Delta I_{\text {ped }}$ is plotted against $\log t_{i}$. For $k^{\prime}>0$ the curve will be flat for values of $t_{i}$ not much less than $\tau$, the absolute value of the slope increasing as $\log t_{i}$ decreases, with an asymptotic value of -1 for $t_{i}$ very small. The larger $k^{\prime}$ the more marked the departure from Bloch's law would be.

When Eq. 31 applies, if $t_{i} \geqslant \tau$ the equation can be written as $\Delta I_{\text {ped }}=k / t^{-1 / 2}$, giving a slope of -0.5 on a $\log \cdot \log$ plot. For $t_{i}<\tau$, when $I_{N}=0$ (the pulsed FCP case) we have a slope of -0.5 for all values of $t_{i}$; when $I_{p}=0$ (the continuous FCP case) we have a slope of -1 ; when both $I_{N}$ and $I_{p}$ are greater than zero, as $t_{i}$ decreases we have a transition from a slope of -0.5 to an asymptotic value of -1 .

Variation in $\boldsymbol{A}_{i}$. This gives analogous predictions.

Variation in $I$. If we vary $I_{N}$ with $I_{p}$ held constant, when Eq. 31 applies, if $A_{i} t_{i} I_{p} \gg A_{r} t_{r} I_{N}$ then $\Delta I_{p e d}$ will at first increase very slowly as $I_{N}$ increases, giving a slope, on log-log coordinates, not much greater than 0 . As $I_{\mathbf{N}}$ increases the slope will increase towards an asymptote of 0.5 . When Eq. 32 holds, if $\mathbf{A}_{\mathbf{i}} \mathbf{t}_{\mathbf{i}} \mathbf{I}_{\mathbf{p}} \gg \mathbf{A}_{\mathbf{r}} \mathbf{t}_{\mathbf{T}} \mathbf{I}_{\mathbf{N}}$ the slope of $\log \Delta \mathbf{I}_{\text {ped }}$ against $\log I_{N}$ will again be low, increasing as $I_{N}$ increases, to an asymptote of 1 . An identical set of predictions can also be derived for the "I $I_{p}$ function" that would be given if $\log \Delta \mathrm{l}_{\text {ped }}$ were plotted against $\log I_{p}$ with $I_{N}$ constant. All these predictions must be qualified, however, by the consideration that when $I_{p}$ is small as compared with $I_{N}$ the eye will be well adapted and these equations will apply as they stand; but when $I_{p}$ is relatively large the situation will be closer to that obtaining in the pulsed FCP: light adaptation will be relatively inadequate and $\Delta \mathrm{I}_{\text {ped }}$ will increase more rapidly than would otherwise be predicted, for the reasons discussed in Section IV.

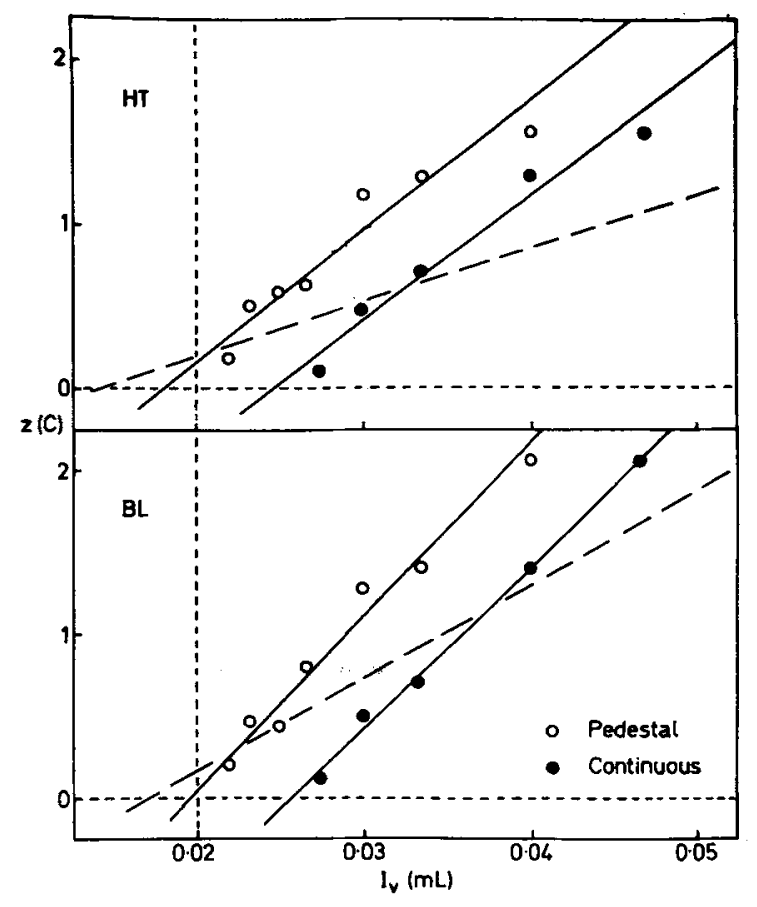

Fig. 10. Results obtained by Leshowitz, Taub, and Raab (1968) for Ss BL and HT. Data are shown for the continuous (filled symbols) and pedestal (open symbols) FCPs with the best-fitting lines given by probit analysis, and for the pulsed FCP the best-fitting line is shown alone as a dashed line. In each case $\log I_{N}=\overline{2} .3 \log \mathrm{mL}$ and $t_{i}=32 \mathrm{msec}$, and in the pedestal procedure $\log I_{p}=\overline{2} .3 \log \mathrm{mL} . \mathrm{z}(\mathrm{C})$ is the standardized normal deviate corresponding to $P(C)$. The coordinates shown in dotted lines correspond to $I_{v}=I_{N}\left(\Delta I_{v}=0\right)$ and $z(C)=0$.

Data against which these predictions could be tested are not yet available, but other predictions can be derived from the equations above for which relevant data exist:

(1) Comparison of $\Delta I_{\text {ped }}$ and $\Delta I_{\text {cfc }}$ for the same $I_{N}$ : (a) We first derive predictions on the assumption that differencing is the strategy employed in the continuous FCP. Let us compare Eq. 30 with Eq. 19. If $I_{N}$ has the same value in each case, and $I_{p}$ is added in Eq. 30, we can evidently predict (i) $\Delta \mathrm{I}_{\text {ped }}$ will be greater than

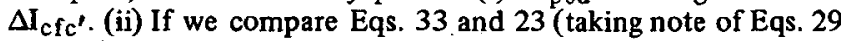
and 18) it is also evident that the slope of the psychometric function will be steeper for the continuous FCP than when the pedestal is added. If light adaptation is imperfect in the pedestal procedure this will tend to reinforce both predictions. (b) Let us assume that the double detection strategy is employed in the continuous FCP. We then compare Eq. 30 with Eq. 3. If we take Eq. 3 as giving an estimate of $\Delta I_{\mathrm{cfc}}{ }^{\prime \prime}$, we see that the two equations differ in two ways. Firstly, $z$ in Eq. 3, which may be of the order $2-6$, corresponds to $0.9539\left(1-\mathrm{r}_{\mathrm{E}}\right)^{1 / 2} \leqslant 1.35$ in Eq. 30; this difference will tend to make $\Delta \mathrm{I}_{\mathrm{cfc}}$ " greater than $\Delta \mathrm{I}_{\text {ped }}$. Secondly, Eq. 30 includes terms containing $I_{p}$ that will tend to raise $\Delta \mathrm{I}_{\text {ped }}$, as will any deterioration in light adaptation consequent on the use of a pedestal. Thus we can predict: (i) Under some conditions $\Delta \mathrm{I}_{\text {ped }}$ may be less than $\Delta \mathrm{I}_{\mathrm{cfc}}{ }^{\prime \prime}$. (ii) The slope of the pedestal psychometric function is determined by $\sigma_{\text {Iped }}$, given by Eq. 33 . If the $S$ employs a double detection strategy in the continuous FCP, we have seen that the slope of the probit line that might be fitted to the resulting data would depend on $\sigma_{I}$ and on the flattening effect of transposing the normal ogive to the asymptotes $0.5(1-\varepsilon)$ and $0.5(2-\varepsilon)$; this effect will depend on $\mathscr{E}$, which is unknown. From these 
considerations we cannot derive any definite prediction about the relations of the two slopes.

Data relevant to these predictions, obtained by Leshowitz, Taub, and Raab (1968) for two Ss, are shown in Fig. 10. The figure shows data for the pedestal and continuous procedures, with $\log \mathrm{I}_{\mathrm{N}}=\overline{2} .3 \log \mathrm{mL}$, and $\mathrm{t}_{\mathrm{i}}=32 \mathrm{msec}$, in each case, and with a pedestal of intensity $\overline{2} .3 \log \mathrm{mL}$ added in the pedestal procedure. The best-fitting lines given by probit analysis are also shown. The most striking effect is that the threshold for the pedestal procedure is markedly lower than that for the continuous procedure in each case. (The $75 \%$ threshold is the value $\left(I_{v}-I_{N}\right)$ corresponding to $z(C)=0.6745$. . This is contrary to what was predicted if the $S$ employed differencing in the continuous procedure, but in agreement with the prediction for double detection. The slopes are similar in the two cases, being slightly flatter, but not significantly so, in the continuous procedure. This again is not in accord with the prediction for differencing.

Leshowitz, Taub, and Raab (1968) report a more shallow slope for the pedestal than for the continuous procedure. This is not in disagreement with the present description, as they plotted the data against $\log I_{v}$, not $I_{v}$ as here.

These results strongly support the evidence obtained in the previous section that Ss may employ double detection rather than the differencing strategy in the continuous procedure.

The dashed line is the best-fitting line for the pulsed FCP with the same parameters, in each case (curve $c$ in Fig. 6 for BL, and curve $a$ in Fig. 7 for HT). The discussion up to this point suggests the following overall explanation for the relations between the three procedures shown in Fig. 10. In the continuous FCP the $S$ prefers the double detection strategy. In the pedestal procedure he is forced to adopt the differencing strategy; with these parameters its greater sensitivity more than compensates for the increased central variance due to $I_{p}$, and for any impairment in light adaptation. Thus "negative masking" (Leshowitz, Taub, \& Raab, 1968), a fall in threshold consequent on the addition of $I_{p}$, results from a forced change in decision strategy, rather than from any peripheral sensory effect of the pedestal. The pulsed procedure does not have the continuous $\overline{2} .3 \log \mathrm{mL}$ background illumination that is present in the pedestal procedure. The advantage in reduced overall intensity is far outweighed by the resulting disadvantage of inadequate light adaptation, here apparently a much greater disadvantage than in the pedestal procedure, with the result that the psychometric function is flatter, and the $75 \%$ threshold correspondingly higher than in the pedestal procedure. (It is possible that the absence of adequate light adaptation impairs performance more for the higher values of $I_{v}$, as was suggested above. If so, this would contribute to making the line fitted to the pulsed FCP appear flat.)

(2) The effect of variation in $I_{p}$. If we add a pedestal to the continuous FCP, what will the effect on threshold be as a function of the magnitude of $I_{p}$ ? If the $S$ employs the double detection strategy in the continuous FCP, then when the added pedestal is sufficiently large he will switch to differencing, the strategy appropriate to the pedestal procedure, and if parameters such as those in Fig. 10 apply, the threshold will fall (or if $\Delta \mathrm{I}_{\mathrm{v}}$ is held constant, $P(C)$ will rise).

What will happen if $I_{p}$ is small? In the discussion of the double detection strategy, we assumed that if a detection occurred at locus $a$ or at $b$ the $S$ would respond accordingly; but if detections occurred at both or at neither he would guess. When double detections occur frequently this becomes unsatisfactory and the $S$ might then switch strategies as a response to this. However, a more attractive idea is that he normally applies a "combined" strategy: when a detection occurs at $a$ or at $b$ he responds in accordance with the double detection strategy, but when

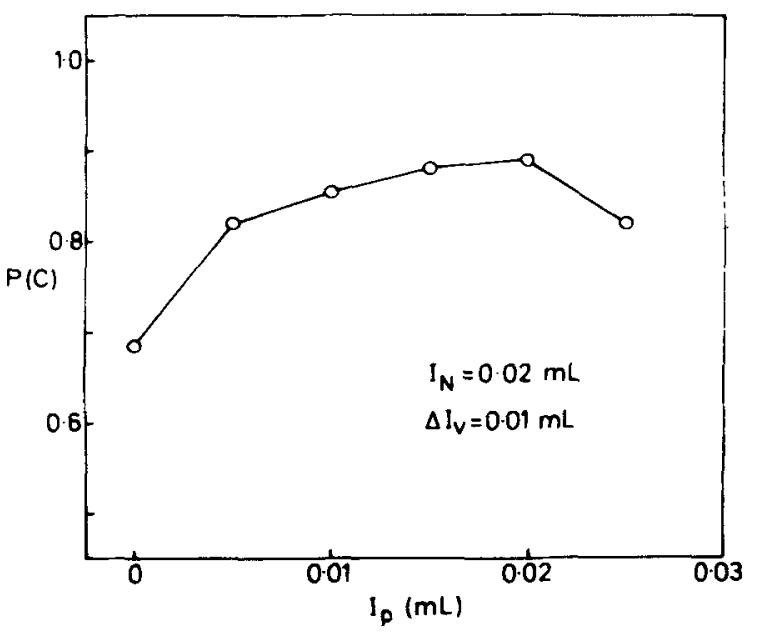

Fig. 11. Values of $P(C)$ for $\log I_{N}$ constant at $\overline{2} .3 \log \mathrm{mL}$, $\log \Delta I_{v}$ constant at $\overline{2} .0 \log \mathrm{mL}$, and $I_{p}$ varied; means of the data for two Ss who gave similar results (Leshowitz, Taub, \& Raab, 1968).

detections occur at both or neither his response is determined by the difference between the two central effects. When double detections are infrequent the results of the combined strategy would be very similar to the double detection strategy as we have described it. But if $I_{p}$ is increased gradually above 0 , the proportion of trials giving two detections and so resulting in the application of differencing will increase smoothly, reaching a maximum when $I_{p}$ is always detectable. Thus, as $I_{p}$ is increased above 0 , there should first be a continuous fall in threshold [or rise in $P(C)$ for a fixed value of $\left.I_{v}\right]$. When differencing is fully established, further increase in $I_{p}$ should result in a rise in threshold [or fall in $P(C)$ ], in accordance with Eq. 30.

Figure 11 shows data relevant to this prediction (Leshowitz, Taub, \& Raab, 1968). The background was kept at a fixed value of $\overline{2} .3 \log \mathrm{mL}, \log \Delta \mathrm{I}_{\mathrm{v}}$ was kept fixed at $\overline{2} .0 \log \mathrm{mL}$, and $\mathrm{P}(\mathrm{C})$ was determined for a series of values of $I_{p}$. As $I_{p}$ increases there is a smooth increase in $P(C)$ to a peak at $\log I_{p}=\overrightarrow{2} .3 \log \mathrm{mL}$. This is an appropriate value, as the mean of $\Delta \mathbf{I}_{\mathrm{cfc}}$ for $\mathrm{I}_{\mathrm{N}}=\overline{2} .3 \mathrm{log} \mathrm{mL}$ for these two Ss was $\overline{2} .15 \log \mathrm{mL}$ (see Fig. 4), so that at the peak in Fig. 11, $I_{p}$ must itself be detectable on most or all trials. The next rise in $I_{p}$ produces the fall in $P(C)$ which was predicted for high values of $I_{p}$.

\section{CONCLUSION}

We have outlined a model for visual discrimination that has previously been found to predict the laws of spatial and temporal summation and the form of the Weber function for traditional detection procedures, such as the method of constant stimuli. The model was then extended to the forced-choice procedures. When this is done it predicts departures from the classical laws of spatial and temporal summation and an increase in the slope of the Weber function for the pulsed forced-choice procedure. Data are presented for temporal summation and the Weber function that agree with these novel predictions. Data obtained with the continuous forced-choice procedure are examined. Predictions made by the model are also confirmed in this case. Two strategies that the S may follow are distinguished, and it is shown that the evidence suggests that in the continuous forced-choice procedure (at any rate with the long ISI that applied to the data examined here) the S employs the "double detection strategy," i.e., he either detects or fails to detect an increment at each of the two alternative locations, and rests his final response on this, rather 
than taking the difference between his observations of the two locations (the "differencing strategy"): there is a bias to detect rather than compare. An analysis of the forced-choice procedure with a pedestal is also given, and a comparison of results obtained with this procedure and with the continuous procedure further supports the conclusion that the $S$ employs the double detection strategy in the latter.

\section{REFERENCES}

AGUILAR, M., \& STILES, W. S. Saturation of the rod mechanism of the retina at high levels of stimulation. Optica Acta, 1954, 1, 59-65.

BAKER, H. D. Initial stages of dark and light adaptation. Journal of the Optical Society of America, 1963, 53, 98-103.

BARLOW, H. B. Retinal noise and absolute threshold. Journal of the Optical Society of America, 1956, 46, 634-649.

BARLOW, H. B. Increment thresholds at low intensities considered as signal/noise discriminations. Journal of Physiology, 1957, 136, 469-488.

BARLOW, H. B., FITZHUGH, R., \& KUFFLER, S. W. Change of organization in the receptive fields of the cat's retina during dark adaptation. Journal of Physiology, 1957, 137, 338-354.

BIRREN, J. E., CARDON, P. V., \& PHILLIPS, S. L. Reaction time as a function of the cardiac cycle in young adults. Science, 1963, 140, 195-196.

BLACKWELL, H. R. Neural theories of simple visual discriminations. Journal of the Optical Society of America, 1963, 53, 129-160.

CALLAWAY, E., \& BUCHSBAUM, M. Effects of cardiac and respiratory cycles on averaged visual evoked responses. Electroencephalography \& Clinical Neurophysiology, 1965, 19, 476-480.

COHN, R. Human electroencephalogram and electrocardiogram correlations by periodogram analysis. Nature, $1962,193,888-889$.

CORNSWEET, T. N., \& PINSKER, H. M. Luminance discrimination of brief flashes under various conditions of adaptation. Journal of Physiology, 1965, 176, 294-310.

CRAWFORD, B. H. Visual adaptation in relation to brief conditioning stimuli. Proceedings of the Royal Society, 1947, 134B, 283-302.

FINNEY, D. J. Probit analysis. (2nd ed.) Cambridge: Cambridge University Press, 1952.

GREGORY, R. L. An experimental treatment of vision as an information source and noisy channel. In E. C. Cherry (Ed.), Information theory. Third London Symposium. London: Butterworth, 1956.

HECHT, S., SHLAER, S., \& PIRENNE, M. H. Energy, quanta and vision. Journal of General Physiology, 1942, 25, 819-840.

KELLER, M. The relation between the critical duration and intensity in brightness discrimination. Journal of Experimental Psychology, 1941, $28,407-418$.
LESHOWITZ, B., TAUB, H. B., \& RAAB, D. H. Visual detection of signals in the presence of continuous and pulsed backgrounds. Perception \& Psychophysics, 1968, 4, 207-213.

MCGILL, W. J. Neural counting mechanisms and energy detection in audition. Journal of Mathematical Psychology, 1967, 4, 351-376.

NEYMAN, J., \& PEARSON, E. S. On the problem of the most efficient tests of statistical hypotheses. Philosophical Transactions of the Royal Society, A, 1933, 231, 289-337.

ROSE, A. The sensitivity performance of the human eye on an absolute scale. Journal of the Optical Society of America, 1948, 38, 196-208.

SWETS, J. A., TANNER, W. P., \& BIRDSALL, T. G. Decision processes in perception. Psychological Review, 1961, 68, 301-340.

TANNER, W. P., \& SWETS, J. A. A decision-making theory of visual detection. Psychological Review, 1954, 61, 401-409.

THURSTONE, L. L. Psychophysical analysis. American Journal of Psychology, 1927, 38, 368-389.

TREISMAN, M. Noise and Weber's Law: The discrimination of brightness and other dimensions. Psychological Review, 1964a, 71, 314-330.

TREISMAN, M. The effect of one stimulus on the threshold for another: An application of signal detectability theory. British Journal of Statistical Psychology, 1964b, 17, 15-35.

TREISMAN, M. Signal detection theory and Crozier's law: Derivation of a new sensory scaling procedure. Journal of Mathematical Psychology, $1965,2,205-218$.

TREISMAN, M. A statistical decision model for sensory discrimination which predicts Weber's law and other sensory laws: Some results of a computer simulation. Perception \& Psychophysics, 1966, 1, 203-230.

WERTHEIMER, M. An investigation of the "randomness" of threshold measurements. Journal of Experimental Psychology, 1953, 45, 294-303.

WETHERILL, G. B. \& LEVITT, H. Sequential estimation of points on a psychometric function. British Journal of Mathematical \& Statistical Psychology, 1965, 18, 1-10.

\section{NOTES}

1. The work reported here was supported by a grant to M. Treisman from the Medical Research Council. The paper was prepared while B. Leshowitz was a U.S. Public Health Service predoctoral fellow and a member of Dr. David H. Raab's laboratory at Brooklyn College. We would like to thank Dr. Cornsweet for generously making his data available.

2. Address: Department of Psychology, University of Reading, Building 3, Earley Gate, Whiteknights, Reading, England.

3. Present address: Department of Psychology, University of California at San Diego, La Jolla, California 92037.

(Accepted for publication March 13, 1969.) 